\title{
Parameter Identification and Hybrid Synchronization in an Array of Coupled Chaotic Systems with Ring Connection: An Adaptive Integral Sliding Mode Approach
}

\author{
Nazam Siddique $(\mathbb{D}$ and Fazal ur Rehman $(\mathbb{D})$ \\ Department of Electrical Engineering, Capital University of Science and Technology, Islamabad, Pakistan \\ Correspondence should be addressed to Nazam Siddique; nazim.siddiq@gmail.com
}

Received 10 August 2017; Revised 11 October 2017; Accepted 28 November 2017; Published 30 January 2018

Academic Editor: Ton D. Do

Copyright @ 2018 Nazam Siddique and Fazal ur Rehman. This is an open access article distributed under the Creative Commons Attribution License, which permits unrestricted use, distribution, and reproduction in any medium, provided the original work is properly cited.

\begin{abstract}
This article presents an adaptive integral sliding mode control (SMC) design method for parameter identification and hybrid synchronization of chaotic systems connected in ring topology. To employ the adaptive integral sliding mode control, the error system is transformed into a special structure containing nominal part and some unknown terms. The unknown terms are computed adaptively. Then the error system is stabilized using integral sliding mode control. The controller of the error system is created that contains both the nominal control and the compensator control. The adapted laws and compensator controller are derived using Lyapunov stability theory. The effectiveness of the proposed technique is validated through numerical examples.
\end{abstract}

\section{Introduction}

Ever since the classic effort by Pecora and Carroll [1], the synchronization in chaotic systems became an active research area because of its useful applications [2-5]. Different synchronization schemes for chaotic systems were studied and investigated [6-10]. Among these schemes, hybrid synchronization [11-19] is one in which some of the chaotic systems are synchronized whereas others are antisynchronized. Due to its importance, hybrid synchronization has been the subject of many research works. These works include study of synchronization/antisynchronization for permanent magnet synchronous motors connected in ring topology [11], function projective type synchronization for complex dynamical networks [12], investigation of complete synchronization and antiphase synchronization together [13], hybrid synchronization of networks having heterogeneous systems [14], study of synchronization for fractional-order systems [15], investigation of synchronization for systems with hyperchaotic nature $[16,17]$, and study of synchronization for complex networks $[18,19]$. Study of hybrid synchronization involves multiple chaotic systems like complete, adaptive, global, projective, and antisynchronization systems [17, 20-27], and synchronization systems in multiple coupled complex networks [28, 29]. Currently, hybrid synchronization of several connected chaotic systems is a hot topic of research and the work includes investigation of complex network synchronization for perturbations and delays [30] and a study of neural networks for synchronization [31].

In this paper, chaotic systems connected in ring topology are considered which can be shown in Figure 1. This arrangement was studied in [32] for the known parameter case. We extend this work and consider that all the parameters of all the systems in the ring connected network are unknown. To reach hybrid synchronization in this system we use adaptive integral SMC.

SMC is a distinct nonlinear control method. The objective of the SMC method is to drive the states of the system to a specific surface, called sliding manifold. When the surface is touched, the dynamic system is required to persist on it afterward. The main disadvantage of SMC is discontinuous control law. In practice, this creeps towards an undesirable occurrence called "chattering." The closed-loop dynamics of the system in SMC depend only on the design parameters of 


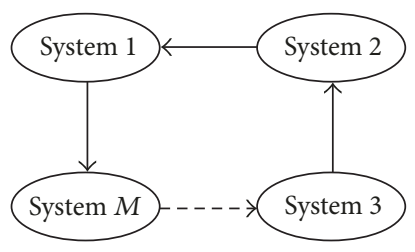

FIGURE 1: Chaotic systems connected in ring topology [32].

the switching sliding manifold. Sliding mode control also offers several benefits like simplicity, robustness to external disturbance, and parameter variation and quick response. The integral SMC, a variant of SMC, guarantees the robustness [33] because of eliminating the reaching-phase. Since reaching-phase is eradicated therefore robustness of the dynamic system can be sure all the way through the system response, beginning from initial conditions. The integral sliding mode control combines both the nominal control which steadies the nominal system and the discontinuous control which discards uncertainties.

In this research, we use adaptive integral sliding mode control technique to identify the unknown parameters and to achieve the hybrid synchronization of many chaotic systems connected in the ring topology. By using this technique, the error system is transformed into a special structure containing nominal part and some unknown terms. The unknown terms are computed adaptively. Then the error system is synthesized using integral sliding mode control. The controller of the error system is created that contains both the nominal control and the compensator control. The adaptive laws and compensator controller are derived using Lyapunov stability theory. The effectiveness of the proposed technique is validated through numerical examples.

The remainder of the paper is arranged as follows; Section 2 presents preliminaries and system description. Section 3 presents proposed control strategies for the general case of hybrid synchronization. Section 4 presents application examples. Section 5 presents discussion and simulation results and in the last section, paper is concluded.

\section{System Description and Preliminaries}

The structure of chaotic systems connected in ring topology is described as

$$
\begin{gathered}
\dot{x}_{1}=f_{1}\left(x_{1}\right)+F_{1}\left(x_{1}\right) \theta_{1}+D_{1}\left(x_{M}-x_{1}\right) \\
\dot{x}_{2}=f_{2}\left(x_{2}\right)+F_{2}\left(x_{2}\right) \theta_{2}+D_{2}\left(x_{1}-x_{2}\right) \\
\vdots \\
\dot{x}_{M}=f_{M}\left(x_{M}\right)+F_{M}\left(x_{M}\right) \theta_{M}+D_{M}\left(x_{M-1}-x_{M}\right),
\end{gathered}
$$

where $x_{1}, x_{2}, \ldots, x_{M}$ are vectors and $x_{o}=\left(x_{o 1}, x_{o 2}, \ldots\right.$, $\left.x_{o M}\right)^{T}, f_{o}: \mathfrak{R}^{m} \rightarrow \mathfrak{R}^{m}, o=1,2, \ldots, M$ are the nonlinear continuous function, and $\theta_{o} \in \mathfrak{R}^{g}, o=1,2,3,4, \ldots, M$ are vectors of unknown parameters. $F_{o}\left(x_{o}\right) \in \Re^{m \times g}, o=1,2$, $3,4, \ldots, M$ are matrices, $D_{o}=\operatorname{diag}\left\{d_{o 1}, d_{o 2}, \ldots, d_{o M}\right\}, o=$ $1,2,3,4, \ldots, M$ are $m$ dimensional diagonal matrices, and $d_{o p} \geq 0$ represent diagonal matrix parameters. When $f_{o}(\cdot) \neq$ $f_{p}(\cdot), o, p=1,2,3,4, \ldots, M$, then the above arrangement has nonidentical systems.

Remark 1. In (1), all the chaotic systems are connected in ring topology [17], because the first system is connected with $M$ th and second is connected with first; and so the final chaotic system is connected with $M-1$ system.

So the hybrid synchronization for the above system (1) may be expressed as follows:

$$
\begin{gathered}
\dot{x}_{1}=f_{1}\left(x_{1}\right)+F_{1}\left(x_{1}\right) \theta_{1}+D_{1}\left(x_{M}-x_{1}\right) \\
\dot{x}_{2}=f_{2}\left(x_{2}\right)+F_{2}\left(x_{2}\right) \theta_{2}+D_{2}\left(x_{1}-x_{2}\right)+u_{1} \\
\vdots \\
\dot{x}_{M}=f_{M}\left(x_{M}\right)+F_{M}\left(x_{M}\right) \theta_{M}+D_{M}\left(x_{M-1}-x_{M}\right) \\
+u_{M-1} .
\end{gathered}
$$

Definition 2. For system (2), we define that it exhibits hybrid synchronization if controllers $u_{o}, o=1,2,3,4, \ldots, M-$ 1 exist such that all trajectories $x_{1}(t), x_{2}(t), x_{3}(t), x_{4}(t), \ldots$, $x_{M}(t)$ for any initial condition $\left(x_{1}(0), x_{2}(0), x_{3}(0), x_{4}(0)\right.$, $\left.\ldots, x_{M}(0)\right)$ satisfy the following:

(1) For the antisynchronization errors $e_{r o}=\left(e_{r o 1}, e_{r o 2}\right.$, $\left.\ldots, e_{\text {rom }}\right)^{T}, o=1,2,3,4, \ldots, M-1$, we have that

$$
\begin{aligned}
\lim _{t \rightarrow \infty}\left\|e_{r o}\right\|=\lim _{t \rightarrow \infty}\left\|x_{o}(t)+x_{o+1}(t)\right\| & =0, \\
o & =1,2,3,4, \ldots, M-1 .
\end{aligned}
$$

(2) The Synchronization errors $e_{r p}^{\prime}=\left(e_{r p 1}^{\prime}, e_{r p 2}^{\prime}, \ldots\right.$, $\left.e_{r p m}^{\prime}\right)^{T}$ and $e_{r q}^{\prime}=\left(e_{r q 1}^{\prime}, e_{r q 2}^{\prime}, \ldots, e_{r q m}^{\prime}\right)^{T}$ if $M(M \geq 3)$ is odd; then $e_{r p}^{\prime}$ and $e_{r q}^{\prime}$ satisfy

$$
\begin{array}{r}
\lim _{t \rightarrow \infty}\left\|e_{r p}^{\prime}\right\|=\lim _{t \rightarrow \infty}\left\|x_{p+2}(t)-x_{p}(t)\right\|=0, \\
p=1,3,5,7,9, \ldots, M-2 \\
\lim _{t \rightarrow \infty}\left\|e_{r q}^{\prime}\right\|=\lim _{t \rightarrow \infty}\left\|x_{q+2}(t)-x_{q}(t)\right\|=0, \\
q=2,4,6,8,10, \ldots, M-3
\end{array}
$$

and if $M(M \geq 4)$ is even, then $e_{r p}^{\prime}$ and $e_{r q}^{\prime}$ satisfy

$$
\begin{array}{r}
\lim _{t \rightarrow \infty}\left\|e_{r p}^{\prime}\right\|=\lim _{t \rightarrow \infty}\left\|x_{p+2}(t)-x_{p}(t)\right\|=0, \\
p=1,3,5,7,9, \ldots, M-3 \\
\lim _{t \rightarrow \infty}\left\|e_{r q}^{\prime}\right\|=\lim _{t \rightarrow \infty}\left\|x_{q+2}(t)-x_{q}(t)\right\|=0, \\
q=2,4,6,8,10, \ldots, M-2 .
\end{array}
$$

We notice from this definition that the error systems $e_{r o}, e_{r p}^{\prime}$, and $e_{r q}^{\prime}$ are globally and asymptotically stable. This shows that, to ensure hybrid synchronization, we have to design controllers $u_{o}(t)$ to make $e_{r o}, e_{r p}^{\prime}$, and $e_{r q}^{\prime}$ converge to zero. 


\section{Parameter Identification and Hybrid Synchronization for the General Case}

For antisynchronization, the error vectors are

$$
\begin{aligned}
& e_{r 1}=x_{2}+x_{1} \\
& e_{r 2}=x_{3}+x_{2}, \ldots, e_{M-1}=x_{M}+x_{M-1} .
\end{aligned}
$$

Let $\hat{\theta}_{o}$ be estimates of $\theta_{o}, o=1,2,3,4, \ldots, M$, respectively, and let $\tilde{\theta}_{o}=\theta_{o}-\hat{\theta}_{o}, o=1,2,3,4, \ldots, M$ be errors while estimating the parameters $\theta_{o}, o=1,2, \ldots, M$, respectively. Derivative of (6) leads to the following:

$$
\begin{aligned}
\dot{e}_{r 1} & =\dot{x}_{2}+\dot{x}_{1}=f_{2}\left(x_{2}\right)+F_{2}\left(x_{2}\right) \hat{\theta}_{2}+F_{2}\left(x_{2}\right) \tilde{\theta}_{2} \\
& +D_{2}\left(x_{1}-x_{2}\right)+u_{1}+f_{1}\left(x_{1}\right)+F_{1}\left(x_{1}\right) \hat{\theta}_{1} \\
& +F_{1}\left(x_{1}\right) \tilde{\theta}_{1}+D_{1}\left(x_{M}-x_{1}\right) \\
\dot{e}_{r 2} & =\dot{x}_{3}+\dot{x}_{2}=f_{3}\left(x_{3}\right)+F_{3}\left(x_{3}\right) \hat{\theta}_{3}+F_{3}\left(x_{3}\right) \tilde{\theta}_{3} \\
& +D_{3}\left(x_{2}-x_{3}\right)+u_{2}+f_{2}\left(x_{2}\right)+F_{2}\left(x_{2}\right) \hat{\theta}_{2}
\end{aligned}
$$

$$
\begin{aligned}
& +F_{2}\left(x_{2}\right) \tilde{\theta}_{2}+D_{2}\left(x_{1}-x_{2}\right)+u_{1} \\
\dot{e}_{r 3} & =\dot{x}_{4}+\dot{x}_{3}=f_{4}\left(x_{4}\right)+F_{4}\left(x_{4}\right) \hat{\theta}_{4}+F_{4}\left(x_{4}\right) \tilde{\theta}_{4} \\
& +D_{4}\left(x_{3}-x_{4}\right)+u_{3}+f_{3}\left(x_{3}\right)+F_{3}\left(x_{3}\right) \hat{\theta}_{3} \\
& +F_{3}\left(x_{3}\right) \tilde{\theta}_{3}+D_{3}\left(x_{2}-x_{3}\right)+u_{2}
\end{aligned}
$$

$$
\begin{aligned}
& \dot{e}_{r M-1}=\dot{x}_{M}+q \dot{x}_{M-1}=f_{M}\left(x_{M}\right)+F_{M}\left(x_{M}\right) \hat{\theta}_{M} \\
& \quad+F_{M}\left(x_{M}\right) \tilde{\theta}_{M}+D_{M}\left(x_{M-1}-x_{M}\right)+u_{M-1} \\
& \quad+f_{M-1}\left(x_{M-1}\right)+F_{M-1}\left(x_{M-1}\right) \widehat{\theta}_{M-1} \\
& \quad+F_{M-1}\left(x_{M-1}\right) \tilde{\theta}_{M-1}+D_{M-1}\left(x_{M-2}-x_{M-1}\right) \\
& \quad+u_{M-2}
\end{aligned}
$$

which can be written as

$\left[\begin{array}{c}\dot{e}_{r 1} \\ \dot{e}_{r 2} \\ \vdots \\ \dot{e}_{r M-1}\end{array}\right]$

$=\left[\begin{array}{c}f_{2}\left(x_{2}\right)+f_{1}\left(x_{1}\right)+F_{2}\left(x_{2}\right) \hat{\theta}_{2}+F_{1}\left(x_{1}\right) \hat{\theta}_{1}+D_{2}\left(x_{1}-x_{2}\right)+D_{1}\left(x_{M}-x_{1}\right) \\ f_{3}\left(x_{3}\right)+f_{2}\left(x_{2}\right)+F_{3}\left(x_{3}\right) \hat{\theta}_{3}+F_{2}\left(x_{2}\right) \hat{\theta}_{2}+D_{3}\left(x_{2}-x_{3}\right)+D_{2}\left(x_{1}-x_{2}\right) \\ \vdots \\ f_{M}\left(x_{M}\right)+f_{M-1}\left(x_{M-1}\right)+F_{M}\left(x_{M}\right) \hat{\theta}_{M}+F_{M-1}\left(x_{M-1}\right) \hat{\theta}_{M-1}+D_{M}\left(x_{M-1}-x_{M}\right)+D_{M-1}\left(x_{M-2}-x_{M-1}\right)\end{array}\right]$

$$
+\left[\begin{array}{c}
F_{2}\left(x_{2}\right) \tilde{\theta}_{2}+F_{1}\left(x_{1}\right) \tilde{\theta}_{1} \\
F_{3}\left(x_{3}\right) \tilde{\theta}+F_{2}\left(x_{2}\right) \tilde{\theta}_{2} \\
\vdots \\
F_{M}\left(x_{M}\right) \tilde{\theta}_{M}+F_{M-1}\left(x_{M-1}\right) \tilde{\theta}_{M-1}
\end{array}\right]+\left[\begin{array}{ccccc}
1 & 0 & 0 & \cdots & 0 \\
1 & 1 & 0 & \cdots & 0 \\
0 & 1 & 1 & \cdots & 0 \\
\vdots & \vdots & \vdots & \vdots & \vdots \\
0 & 0 & \cdots & 1 & 1
\end{array}\right]\left[\begin{array}{c}
u_{1} \\
u_{2} \\
\vdots \\
u_{M-1}
\end{array}\right] .
$$

By choosing

$$
\left[\begin{array}{c}
u_{1} \\
u_{2} \\
\vdots \\
u_{M-1}
\end{array}\right]=\left[\begin{array}{ccccc}
1 & 0 & 0 & \cdots & 0 \\
1 & 1 & 0 & \cdots & 0 \\
0 & 1 & 1 & \cdots & 0 \\
\vdots & \vdots & \vdots & \vdots & \vdots \\
0 & 0 & \cdots & 1 & 1
\end{array}\right]^{-1}\left\{+\left[\begin{array}{c}
e_{r 2} \\
e_{r 3} \\
\vdots \\
e_{r M-1} \\
v
\end{array}\right]-A\right\}
$$


where $v$ is the new input vector and

$$
A=\left[\begin{array}{c}
f_{2}\left(x_{2}\right)+f_{1}\left(x_{1}\right)+F_{2}\left(x_{2}\right) \hat{\theta}_{2}+F_{1}\left(x_{1}\right) \hat{\theta}_{1}+D_{2}\left(x_{1}-x_{2}\right)+D_{1}\left(x_{M}-x_{1}\right) \\
f_{3}\left(x_{3}\right)+f_{2}\left(x_{2}\right)+F_{3}\left(x_{3}\right) \hat{\theta}_{3}+F_{2}\left(x_{2}\right) \hat{\theta}_{2}+D_{3}\left(x_{2}-x_{3}\right)+D_{2}\left(x_{1}-x_{2}\right) \\
\vdots \\
f_{M}\left(x_{M}\right)+f_{M-1}\left(x_{M-1}\right)+F_{M}\left(x_{M}\right) \hat{\theta}_{M}+F_{M-1}\left(x_{M-1}\right) \hat{\theta}_{M-1}+D_{M}\left(x_{M-1}-x_{M}\right)+D_{M-1}\left(x_{M-2}-x_{M-1}\right)
\end{array}\right]
$$

then system (7) becomes

$$
\begin{gathered}
\dot{e}_{r 1}=e_{r 2}+F_{2}\left(x_{2}\right) \tilde{\theta}_{2}+F_{1}\left(x_{1}\right) \widetilde{\theta}_{1} \\
\dot{e}_{r 2}=e_{r 3}+F_{3}\left(x_{3}\right) \tilde{\theta}_{3}+F_{2}\left(x_{2}\right) \tilde{\theta}_{2} \\
\dot{e}_{r 3}=e_{r 4}+F_{4}\left(x_{4}\right) \tilde{\theta}_{4}+F_{3}\left(x_{3}\right) \widetilde{\theta}_{3} \\
\vdots \\
\dot{e}_{r M-2}=e_{r M-1}+F_{M-1}\left(x_{M-1}\right) \widetilde{\theta}_{M-1} \\
+F_{M-2}\left(x_{M-2}\right) \widetilde{\theta}_{M-2} \\
\dot{e}_{r M-1}=v+F_{M}\left(x_{M}\right) \tilde{\theta}_{M}+F_{M-1}\left(x_{M-1}\right) \widetilde{\theta}_{M-1} .
\end{gathered}
$$

To employ the integral sliding mode control, choose the nominal system for (11) as

$$
\begin{gathered}
\dot{e}_{r 1}=e_{r 2} \\
\dot{e}_{r 2}=e_{r 3} \\
\dot{e}_{r 3}=e_{r 4} \\
\vdots \\
\dot{e}_{r M-2}=e_{r M-1} \\
\dot{e}_{r M-1}=v_{0} .
\end{gathered}
$$

To stabilize the system (11), we choose the Hurwitz sliding surface for system (12) as $\sigma_{0}=(1+d / d t)^{M-2} e_{r 1}=e_{r 1}+c_{1} e_{r 2}+$ $\cdots+c_{M-3} e_{r M-2}+e_{r M-1}$, where the coefficients $c_{o}$ are chosen in such a way that $\sigma_{0}$ becomes Hurwitz polynomial. Then $\dot{\sigma}_{0}=$ $e_{r 2}+c_{1} e_{r 3}+c_{2} e_{r 4}+\cdots+c_{M-3} e_{r M-1}+v_{0}$.

By choosing $v_{0}=-e_{r 2}-c_{1} e_{r 3}-c_{2} e_{r 4}-\cdots-c_{M-3} e_{r M-1}-k \sigma_{0}-$ $k \operatorname{sign}\left(\sigma_{0}\right), k>0$, we have $\dot{\sigma}_{0}=-k \operatorname{sign}\left(\sigma_{0}\right)-k \sigma_{0}$; therefore $\sigma_{0} \rightarrow 0$, which gives $e_{r 1}, e_{r 2}, \ldots, e_{r M-1} \rightarrow 0$. Therefore, system (12) becomes asymptotically stable.

The sliding surface for system (9) is $\sigma=\sigma_{0}+z$. The term $z$ in the sliding surface is an integral term computed later. To avoid the reaching-phase, choose $z(0)$ such that $\sigma(0)=0$. By choosing $v=v_{0}+v_{s}$ where $v_{0}$ is the nominal input vector and $v_{s}$ is compensator terms computed later, then the time derivative of the sliding surface becomes as

$$
\begin{aligned}
\dot{\sigma}= & \dot{\sigma}_{0}+\dot{z} \\
= & \dot{e}_{r 1}+c_{1} \dot{e}_{r 2}+c_{2} \dot{e}_{r 3}+c_{3} \dot{e}_{r 4}+\cdots+c_{M-3} \dot{e}_{r M-2} \\
& +\dot{e}_{r M-1}+\dot{z} \\
= & e_{r 2}+c_{1} e_{r 3}+c_{2} e_{r 4}+\cdots+c_{M-3} e_{r M-1}+v_{0}+v_{s}+\dot{z} \\
& +F_{1}\left(x_{1}\right) \widetilde{\theta}_{1}+\left(1+c_{1}\right) F_{2}\left(x_{2}\right) \widetilde{\theta}_{2} \\
& +\left(c_{1}+c_{2}\right) F_{3}\left(x_{3}\right) \widetilde{\theta}_{3}+\left(c_{2}+c_{3}\right) F_{4}\left(x_{4}\right) \tilde{\theta}_{4}+\cdots \\
& +\left(c_{M-4}+c_{M-3}\right) F_{M-2}\left(x_{M-2}\right) \widetilde{\theta}_{M-2} \\
& +\left(c_{M-3}+1\right) F_{M-1}\left(x_{M-1}\right) \widetilde{\theta}_{M-1}+F_{M}\left(x_{M}\right) \widetilde{\theta}_{M} .
\end{aligned}
$$

By choosing a Lyapunov function: $V=(1 / 2)\left\{\sigma^{T} \sigma+\widetilde{\theta}_{1}^{T} \widetilde{\theta}_{1}+\right.$ $\left.\widetilde{\theta}_{2}^{T} \widetilde{\theta}_{2}+\sum_{o=1}^{M-4} \widetilde{\theta}_{o+2}^{T} \widetilde{\theta}_{o+2}+\widetilde{\theta}_{M-1}^{T} \widetilde{\theta}_{M-1}+\widetilde{\theta}_{M}^{T} \widetilde{\theta}_{M}\right\}$, designing the adaptive laws for $\widetilde{\theta}_{o}, \widehat{\theta}_{o}, o=1,2, \ldots, M$ and computing $v_{s}$ such that $\dot{V}<0$.

Theorem 3. Consider a Lyapunov function $V=(1 / 2)\left\{\sigma^{T} \sigma+\right.$ $\left.\widetilde{\theta}_{1}^{T} \widetilde{\theta}_{1}+\widetilde{\theta}_{2}^{T} \widetilde{\theta}_{2}+\sum_{o=1}^{M-4} \widetilde{\theta}_{o+2}^{T} \widetilde{\theta}_{o+2}+\widetilde{\theta}_{M-1}^{T} \widetilde{\theta}_{M-1}+\widetilde{\theta}_{M}^{T} \widetilde{\theta}_{M}\right\}$. Then $\dot{V}<0$ if the adaptive laws for $\widetilde{\theta}_{o}, \widehat{\theta}_{o}, o=1,2,3,4, \ldots, M$ and the value of $v_{s}$ are chosen as

$$
\begin{aligned}
\dot{z} & =-e_{r 2}-\sum_{o=3}^{M-1} c_{o-2} e_{r o}-v_{0}, \\
v_{s} & =-k \sigma \\
\dot{\tilde{\theta}}_{1} & =-F_{1}^{T}\left(x_{1}\right) \sigma-k_{1} \tilde{\theta}_{1}, \\
\dot{\hat{\theta}}_{1} & =-\dot{\tilde{\theta}}_{1} \\
\dot{\tilde{\theta}}_{2} & =-\left(1+c_{1}\right) F_{2}^{T}\left(x_{2}\right) \sigma-k_{2} \widetilde{\theta}_{2}, \\
\dot{\hat{\theta}}_{2} & =-\dot{\tilde{\theta}}_{2} \\
\dot{\tilde{\theta}}_{o+2} & =-\left(c_{o}+c_{o+1}\right) F_{o+2}^{T}\left(x_{o+2}\right) \sigma-k_{o+2} \tilde{\theta}_{o+2}, \\
\dot{\hat{\theta}}_{o+2} & =-\dot{\tilde{\theta}}_{o+2}, \quad o=1, \ldots, M-4
\end{aligned}
$$




$$
\begin{aligned}
\dot{\tilde{\theta}}_{M-1} & =-\left(c_{M-3}+1\right) F_{M-1}^{T}\left(x_{M-1}\right) \sigma-k_{M-1} \widetilde{\theta}_{M-1}, \\
\dot{\hat{\theta}}_{M-1} & =-\dot{\tilde{\theta}}_{M-1} \\
\dot{\tilde{\theta}}_{M} & =-F_{M}^{T}\left(x_{M}\right) \sigma-k_{M} \widetilde{\theta}_{M}, \\
\dot{\hat{\theta}}_{M} & =-\dot{\tilde{\theta}}_{M} .
\end{aligned}
$$

Proof. Since

$$
\begin{aligned}
\dot{V} & =\sigma^{T} \dot{\sigma}+\widetilde{\theta}_{1}^{T} \dot{\tilde{\theta}}_{1}+\widetilde{\theta}_{2}^{T} \dot{\tilde{\theta}}_{2}+\sum_{o=1}^{M-4} \widetilde{\theta}_{o+2}^{T} \dot{\tilde{\theta}}_{o+2}+\widetilde{\theta}_{M-1}^{T} \dot{\tilde{\theta}}_{M-1} \\
& +\widetilde{\theta}_{M}^{T} \dot{\tilde{\theta}}_{M}=\sigma^{T}\left\{e_{r 2}+\sum_{o=3}^{M-1} c_{o-2} e_{r o}+v_{0}+v_{s}+\dot{z}\right\} \\
& +\widetilde{\theta}_{1}^{T}\left\{\dot{\tilde{\theta}}_{1}+F_{1}^{T}\left(x_{1}\right) \sigma\right\}+\widetilde{\theta}_{2}^{T}\left\{\dot{\tilde{\theta}}_{2}\right. \\
& \left.+\left(1+c_{1}\right) F_{2}^{T}\left(x_{2}\right) \sigma\right\} \\
& +\sum_{o=1}^{M-4} \widetilde{\theta}_{o+2}^{T}\left\{\dot{\tilde{\theta}}_{o+2}+\left(c_{o}+c_{o+1}\right) F_{o+2}^{T}\left(x_{o+2}\right) \sigma\right\} \\
& +\widetilde{\theta}_{M-1}^{T}\left\{\dot{\tilde{\theta}}_{M-1}+\left(c_{M-3}+1\right) F_{M-1}^{T}\left(x_{M-1}\right) \sigma\right\} \\
& +\widetilde{\theta}_{M}^{T}\left\{\dot{\tilde{\theta}}_{M}+F_{M}^{T}\left(x_{M}\right) \sigma\right\}
\end{aligned}
$$

by using (14) we have

$$
\dot{V}=-k \sigma^{2}-\sum_{o=1}^{M} k_{o} \widetilde{\theta}_{o}^{T} \widetilde{\theta}_{o}
$$

From this we conclude that $\sigma, \widetilde{\theta}_{o} \rightarrow 0$. Since $\sigma \rightarrow 0$, therefore $e_{o} \rightarrow 0, o=1,2,3, \ldots, M-1$. Thus the antisynchronization is achieved.

The controllers designed for the antisynchronization are used for the complete synchronization. For this we consider two cases:

Case 1. For odd number of systems and $M(M \geq 3)$, synchronization error is expressed like

$$
\begin{aligned}
& e_{r p}^{\prime}(t)=x_{p+2}(t)-x_{p}(t), \\
& \quad p=1,3,5,7,9, \ldots, M-2 \\
& e_{r q}^{\prime}(t)=x_{q+2}(t)-x_{q}(t),
\end{aligned}
$$

$$
q=2,4,6,8,10, \ldots, M-3 .
$$

Then

$$
\begin{gathered}
\lim _{t \rightarrow \infty}\left\|e_{r p}^{\prime}\right\|=\lim _{t \rightarrow \infty}\left\|x_{p+2}(t)-x_{p}(t)\right\| \\
\lim _{t \rightarrow \infty}\left\|x_{p+2}(t)+x_{p+1}(t)-x_{p+1}(t)-x_{p}(t)\right\| \\
\leq \lim _{t \rightarrow \infty}\left\|x_{p+2}(t)+x_{p+1}(t)\right\| \\
\quad+\lim _{t \rightarrow \infty}\left\|x_{p+1}(t)+x_{p}(t)\right\|=0
\end{gathered}
$$

due to antisynchronization.

Thus

$$
\lim _{t \rightarrow \infty}\left\|e_{r p}^{\prime}\right\|=0, \quad p=1,3,5,7,9, \ldots, M-2 .
$$

Similarly

$$
\begin{aligned}
& \lim _{t \rightarrow \infty}\left\|e_{r p}^{\prime}\right\|=\lim _{t \rightarrow \infty}\left\|x_{q+2}(t)-x_{q}(t)\right\| \\
& =\lim _{t \rightarrow \infty}\left\|x_{q+2}(t)+x_{q+1}(t)-x_{q+1}(t)-x_{q}(t)\right\| \\
& \leq \lim _{t \rightarrow \infty}\left\|x_{q+2}(t)+x_{q+1}(t)\right\| \\
& \quad+\lim _{t \rightarrow \infty}\left\|x_{q+1}(t)+x_{q}(t)\right\|=0 \\
& \lim _{t \rightarrow \infty}\left\|e_{r p}^{\prime}\right\|=0, \quad q=2,4,6,8,10, \ldots, M-3 .
\end{aligned}
$$

Case 2. For even number of systems and $M(M \geq 4)$, synchronization error is expressed like

$$
\begin{aligned}
& e_{r p}^{\prime}=x_{p+2}(t)-x_{p}(t), \\
& e_{r q}^{\prime}=x_{q+2}(t)-x_{q}(t) \\
& \lim _{t \rightarrow \infty}\left\|e_{r p}^{\prime}\right\|=\lim _{t \rightarrow \infty}\left\|x_{p+2}(t)-x_{p}(t)\right\| \\
& \quad=\lim _{t \rightarrow \infty}\left\|x_{p+2}(t)+x_{p+1}(t)-x_{p+1}(t)-x_{p}(t)\right\| \\
& \quad \leq \lim _{t \rightarrow \infty}\left\|x_{p+2}(t)+x_{p+1}(t)\right\| \\
& \quad+\lim _{t \rightarrow \infty}\left\|x_{p+1}(t)+x_{p}(t)\right\|=0 \\
& \lim _{t \rightarrow \infty}\left\|e_{r p}^{\prime}\right\|=0, \quad p=1,3,5,7,9, \ldots, M-3 .
\end{aligned}
$$

Similarly

$$
\begin{array}{r}
\lim _{t \rightarrow \infty}\left\|e_{r q}^{\prime}\right\|=\lim _{t \rightarrow \infty}\left\|x_{q+2}(t)-x_{q}(t)\right\|=0, \\
q=2,4,6,8,10, \ldots, M-2 .
\end{array}
$$

So complete synchronization is too achieved.

\section{Application Examples}

To illustrate the control design procedure for reaching the hybrid synchronization behavior, we present two examples with $M=3$ and $M=4$. 
Example $1(M=3)$. Chen chaotic system, Lorenz chaotic system, and Lü chaotic system, in that order, are selected for this numerical example and are expressed below:

$$
\begin{aligned}
& \dot{x}_{11}=-35 x_{11}+35 x_{12}+d_{11}\left(x_{31}-x_{11}\right) \\
& \dot{x}_{12}=-7 x_{11}+28 x_{12}+x_{11} x_{13}+d_{12}\left(x_{32}-x_{12}\right) \\
& \dot{x}_{13}=-3 x_{13}+x_{11} x_{12}+d_{13}\left(x_{33}-x_{13}\right) \\
& \dot{x}_{21}=-36 x_{21}+36 x_{22}+d_{21}\left(x_{11}-x_{21}\right)+u_{11} \\
& \dot{x}_{22}=20 x_{22}-x_{21} x_{23}+d_{22}\left(x_{12}-x_{22}\right)+u_{12} \\
& \dot{x}_{23}=-3 x_{23}+x_{21} x_{22}+d_{23}\left(x_{13}-x_{23}\right)+u_{13} \\
& \dot{x}_{31}=-10 x_{31}+10 x_{32}+d_{31}\left(x_{21}-x_{31}\right)+u_{21} \\
& \dot{x}_{32}=28 x_{31}-x_{32}-x_{31} x_{33}+d_{32}\left(x_{22}-x_{32}\right)+u_{22} \\
& \dot{x}_{33}=-\frac{8}{3} x_{33}+x_{31} x_{32}+d_{33}\left(x_{23}-x_{33}\right)+u_{23} .
\end{aligned}
$$

By assuming that the systems parameters are unknown, we write systems (24) as

$$
\begin{aligned}
& \dot{x}_{11}=a_{1} x_{11}+b_{1} x_{12}+d_{11}\left(x_{31}-x_{11}\right) \\
& \dot{x}_{12}=c_{1} x_{11}+d_{1} x_{12}-x_{11} x_{13}+d_{12}\left(x_{32}-x_{12}\right) \\
& \dot{x}_{13}=e_{1} x_{13}+x_{11} x_{12}+d_{13}\left(x_{33}-x_{13}\right) \\
& \dot{x}_{21}=a_{2} x_{21}+b_{2} x_{22}+d_{21}\left(x_{11}-x_{21}\right)+u_{11} \\
& \dot{x}_{22}=c_{2} x_{22}-x_{21} x_{23}+d_{22}\left(x_{12}-x_{22}\right)+u_{12} \\
& \dot{x}_{23}=e_{2} x_{23}+x_{21} x_{22}+d_{23}\left(x_{13}-x_{23}\right)+u_{13} \\
& \dot{x}_{31}=a_{3} x_{31}+b_{3} x_{32}+d_{31}\left(x_{21}-x_{31}\right)+u_{21} \\
& \dot{x}_{32}=c_{3} x_{31}-x_{32}-x_{31} x_{33}+d_{32}\left(x_{22}-x_{32}\right)+u_{22} \\
& \dot{x}_{33}=e_{3} x_{33}+x_{31} x_{32}+d_{33}\left(x_{23}-x_{33}\right)+u_{23} .
\end{aligned}
$$

Let $\widehat{a}_{o}, \widehat{b}_{o}, \widehat{c}_{o}, \widehat{e}_{r o}, \widehat{d}_{1}, o=1,2,3$ be estimates of $a_{o}, b_{o}, c_{o}, e_{r o}$, $d_{1}, o=1,2,3$, respectively, and let $\widetilde{a}_{o}=a_{o}-\widehat{a}_{o}, \widetilde{b}_{o}=b_{o}-\widehat{b}_{o}$, $\tilde{c}_{o}=c_{o}-\widehat{c}_{o}, \tilde{e}_{r o}=e_{r o}-\widehat{e}_{r o}, \tilde{d}_{1}=d_{1}-\widehat{d}_{1}, o=1,2,3$ be errors in estimation of $a_{o}, b_{o}, c_{o}, e_{o}, d_{1}, o=1,2,3$, respectively.

Defining

$$
\begin{aligned}
& x_{1}=\left[\begin{array}{l}
x_{11} \\
x_{12} \\
x_{13}
\end{array}\right], \\
& x_{2}=\left[\begin{array}{l}
x_{21} \\
x_{22} \\
x_{23}
\end{array}\right], \\
& x_{3}=\left[\begin{array}{l}
x_{31} \\
x_{32} \\
x_{33}
\end{array}\right],
\end{aligned}
$$

$$
\begin{gathered}
u_{1}=\left[\begin{array}{l}
u_{11} \\
u_{12} \\
u_{13}
\end{array}\right], \\
u_{2}=\left[\begin{array}{l}
u_{21} \\
u_{22} \\
u_{23}
\end{array}\right], \\
\widehat{\theta}=\left[\begin{array}{l}
\widehat{a} \\
\widehat{b} \\
\widehat{c} \\
\widehat{d}
\end{array}\right], \\
\tilde{\theta}=\left[\begin{array}{l}
\tilde{a} \\
\tilde{b} \\
\tilde{c} \\
\tilde{d}
\end{array}\right]
\end{gathered}
$$

then the systems (25) in vector form are written as

$$
\begin{aligned}
& \dot{x}_{1}=f_{1}\left(x_{1}\right)+F_{1}\left(x_{1}\right) \hat{\theta}_{1}+F_{1}\left(x_{1}\right) \tilde{\theta}_{1} \\
& \dot{x}_{2}=f_{2}\left(x_{2}\right)+F_{2}\left(x_{2}\right) \hat{\theta}_{2}+F_{2}\left(x_{2}\right) \tilde{\theta}_{2}+u_{1} \\
& \dot{x}_{3}=f_{3}\left(x_{3}\right)+F_{3}\left(x_{3}\right) \hat{\theta}_{3}+F_{3}\left(x_{3}\right) \tilde{\theta}_{3}+u_{2},
\end{aligned}
$$

where

$$
\begin{aligned}
& f_{1}\left(x_{1}\right)= {\left[\begin{array}{c}
d_{11}\left(x_{31}-x_{11}\right) \\
x_{11} x_{13}+d_{12}\left(x_{32}-x_{12}\right) \\
x_{11} x_{12}+d_{13}\left(x_{33}-x_{13}\right)
\end{array}\right], } \\
& F_{1}\left(x_{1}\right)=\left[\begin{array}{ccccc}
x_{11} & x_{12} & 0 & 0 & 0 \\
0 & 0 & \widehat{c}_{1} & \widehat{d}_{1} & 0 \\
0 & 0 & 0 & 0 & x_{13}
\end{array}\right], \\
& \hat{\theta}_{1}=\left[\begin{array}{c}
\widehat{a}_{1} \\
\hat{b}_{1} \\
\widehat{c}_{1} \\
\widehat{d}_{1} \\
\hat{e}_{1}
\end{array}\right], \\
& \tilde{\theta}_{1}=\left[\begin{array}{l}
\tilde{a}_{1} \\
\tilde{b}_{1} \\
\widetilde{c}_{1} \\
\tilde{d}_{1} \\
\tilde{e}_{1}
\end{array}\right],
\end{aligned}
$$




$$
\begin{aligned}
& f_{2}\left(x_{2}\right)=\left[\begin{array}{c}
d_{21}\left(x_{11}-x_{21}\right) \\
-x_{21} x_{23}+d_{22}\left(x_{12}-x_{22}\right) \\
x_{21} x_{22}+d_{23}\left(x_{13}-x_{23}\right)
\end{array}\right], \\
& F_{2}\left(x_{2}\right)=\left[\begin{array}{cccc}
x_{21} & x_{22} & 0 & 0 \\
0 & 0 & x_{22} & 0 \\
0 & 0 & 0 & x_{23}
\end{array}\right],
\end{aligned}
$$$$
\widehat{\theta}_{2}=\left[\begin{array}{l}
\widehat{a}_{2} \\
\widehat{b}_{2} \\
\widehat{c}_{2} \\
\widehat{e}_{2}
\end{array}\right],
$$$$
\tilde{\theta}_{2}=\left[\begin{array}{l}
\tilde{a}_{2} \\
\tilde{b}_{2} \\
\tilde{c}_{2} \\
\tilde{e}_{2}
\end{array}\right],
$$$$
f_{3}\left(x_{3}\right)=\left[\begin{array}{c}
d_{31}\left(x_{21}-x_{31}\right) \\
-x_{32}-x_{31} x_{33}+d_{32}\left(x_{22}-x_{32}\right) \\
x_{31} x_{32}+d_{33}\left(x_{23}-x_{33}\right)
\end{array}\right] \text {, }
$$$$
F_{3}\left(x_{3}\right)=\left[\begin{array}{cccc}
x_{31} & x_{32} & 0 & 0 \\
0 & 0 & x_{31} & 0 \\
0 & 0 & 0 & x_{33}
\end{array}\right] \text {, }
$$$$
\widehat{\theta}_{3}=\left[\begin{array}{c}
\widehat{a}_{3} \\
\widehat{b}_{3} \\
\widehat{c}_{3} \\
\widehat{e}_{3}
\end{array}\right],
$$

By choosing

$$
\begin{aligned}
& {\left[\begin{array}{l}
u_{1} \\
u_{2}
\end{array}\right]=\left[\begin{array}{ll}
1 & 0 \\
1 & 1
\end{array}\right]^{-1}} \\
& \quad \cdot\left\{-\left[\begin{array}{c}
f_{2}\left(x_{2}\right)+F_{2}\left(x_{2}\right) \hat{\theta}_{2}+f_{1}\left(x_{1}\right)+F_{1}\left(x_{1}\right) \hat{\theta}_{1} \\
f_{3}\left(x_{3}\right)+F_{3}\left(x_{3}\right) \hat{\theta}_{3}+F_{2}\left(x_{2}\right) \hat{\theta}_{2}
\end{array}\right]\right. \\
& \left.+\left[\begin{array}{c}
e_{r 2} \\
v
\end{array}\right]\right\},
\end{aligned}
$$

Therefore

$$
\begin{aligned}
{\left[\begin{array}{c}
\dot{e}_{r 1} \\
\dot{e}_{r 2}
\end{array}\right]=} & {\left[\begin{array}{c}
f_{2}\left(x_{2}\right)+F_{2}\left(x_{2}\right) \hat{\theta}_{2}+f_{1}\left(x_{1}\right)+F_{1}\left(x_{1}\right) \hat{\theta}_{1} \\
F_{2}\left(x_{2}\right) \hat{\theta}_{2}+f_{3}\left(x_{3}\right)+F_{3}\left(x_{3}\right) \hat{\theta}_{3}
\end{array}\right] } \\
& +\left[\begin{array}{c}
F_{1}\left(x_{1}\right) \tilde{\theta}_{1}+F_{2}\left(x_{2}\right) \tilde{\theta}_{2} \\
F_{2}\left(x_{2}\right) \tilde{\theta}_{2}+F_{3}\left(x_{3}\right) \tilde{\theta}_{3}
\end{array}\right]+\left[\begin{array}{ll}
1 & 0 \\
1 & 1
\end{array}\right]\left[\begin{array}{l}
u_{1} \\
u_{2}
\end{array}\right] .
\end{aligned}
$$

where $v=\left[\begin{array}{lll}v_{1} & v_{2} & v_{3}\end{array}\right]^{T}$ is the new input vector, then system (31) becomes

$$
\begin{aligned}
& \dot{e}_{r 1}=e_{2}+F_{2}\left(x_{2}\right) \tilde{\theta}_{2}+F_{1}\left(x_{1}\right) \tilde{\theta}_{1} \\
& \dot{e}_{r 2}=v+F_{3}\left(x_{3}\right) \tilde{\theta}_{3}+F_{2}\left(x_{2}\right) \tilde{\theta}_{2} .
\end{aligned}
$$

The nominal system for (33) is

$$
\begin{aligned}
& \dot{e}_{r 1}=e_{2} \\
& \dot{e}_{r 2}=v_{0},
\end{aligned}
$$

where $v_{0}=\left[\begin{array}{lll}v_{01} & v_{02} & v_{03}\end{array}\right]^{T}$ is the nominal input vector.

The sliding surface for nominal system (34) is $\sigma_{0}=e_{r 1}+$ $e_{r 2}$; that is,

$$
\left[\begin{array}{l}
\sigma_{01} \\
\sigma_{02} \\
\sigma_{03}
\end{array}\right]=\left[\begin{array}{l}
e_{r 11}+e_{r 21} \\
e_{r 12}+e_{r 22} \\
e_{r 13}+e_{r 23}
\end{array}\right]
$$

$$
\begin{aligned}
& e_{r 1}=\left[\begin{array}{l}
e_{r 11} \\
e_{r 12} \\
e_{r 13}
\end{array}\right], \\
& e_{r 2}=\left[\begin{array}{l}
e_{r 21} \\
e_{r 22} \\
e_{r 23}
\end{array}\right]
\end{aligned}
$$

The nominal system (34) is asymptotically stable if $v_{0}=-e_{r 2}-$ $k \sigma_{0}-k \operatorname{sign}\left(\sigma_{0}\right), k>0$. The sliding surface for system (33) is $\sigma=\sigma_{0}+z=e_{r 1}+e_{r 2}+z$, where $z=\left[\begin{array}{lll}z_{1} & z_{3} & z_{3}\end{array}\right]^{T}$ is some integral term and $z(0)$ is chosen in such a way that $\sigma(0)=0$. Defining $v=v_{0}+v_{s}$ where $v_{0}=\left[\begin{array}{lll}v_{01} & v_{02} & v_{03}\end{array}\right]^{T}$ is the nominal 
input and $v_{s}=\left[\begin{array}{lll}v_{s 1} & v_{s 2} & v_{s 3}\end{array}\right]^{T}$ is compensator, then system (33) can be rewritten as

$$
\begin{aligned}
& \dot{e}_{r 11}=e_{r 21}+\tilde{a}_{2} x_{21}+\widetilde{b}_{2} x_{22}+\tilde{a}_{1} x_{11}+\widetilde{b}_{1} x_{12} \\
& \dot{e}_{r 12}=e_{r 22}+\widetilde{c}_{2} x_{22}+\widetilde{c}_{1} x_{11}+\widetilde{d}_{1} x_{12} \\
& \dot{e}_{r 13}=e_{r 23}+\widetilde{e}_{r 2} x_{23}+\widetilde{e}_{r 1} x_{13} \\
& \dot{e}_{r 21}=v_{1}+\tilde{a}_{3} x_{31}+\tilde{b}_{3} x_{32}+\tilde{a}_{2} x_{21}+\tilde{b}_{2} x_{22} \\
& \dot{e}_{r 22}=v_{2}+\widetilde{c}_{3} x_{31}+\widetilde{c}_{2} x_{22} \\
& \dot{e}_{r 23}=v_{3}+\widetilde{e}_{r 3} x_{33}+\widetilde{e}_{r 2} x_{23} .
\end{aligned}
$$

Then $\dot{\sigma}=\dot{e}_{r 1}+\dot{e}_{r 2}+\dot{z}$ gives

$$
\begin{aligned}
\dot{\sigma}_{1}= & e_{r 21}+v_{01}+v_{s 1}+\dot{z}_{1}+\widetilde{a}_{1} x_{11}+\widetilde{b}_{1} x_{12}+2 \widetilde{a}_{2} x_{21} \\
& +2 \widetilde{b}_{2} x_{22}+\widetilde{a}_{3} x_{31}+\widetilde{b}_{3} x_{32} \\
\dot{\sigma}_{2}= & e_{r 22}+v_{02}+v_{s 2}+\dot{z}_{2}+\widetilde{c}_{1} x_{11}+\widetilde{d}_{1} x_{12}+2 \widetilde{c}_{2} x_{22} \\
& +\widetilde{c}_{3} x_{31} \\
\dot{\sigma}_{3}= & e_{r 23}+v_{03}+v_{s 3}+\dot{z}_{3}+2 \widetilde{e}_{r 2} x_{23}+\widetilde{e}_{r 1} x_{13}+\widetilde{e}_{r 3} x_{33} .
\end{aligned}
$$

By choosing a Lyapunov function

$$
\begin{aligned}
V & =\frac{1}{2}\left\{\sigma_{1}^{2}+\sigma_{2}^{2}+\sigma_{3}^{2}+\widetilde{a}_{1}^{2}+\widetilde{a}_{2}^{2}+\widetilde{a}_{3}^{2}+\widetilde{b}_{1}^{2}+\widetilde{b}_{2}^{2}+\widetilde{b}_{3}^{2}\right. \\
& \left.+\widetilde{c}_{1}^{2}+\widetilde{c}_{2}^{2}+\widetilde{c}_{3}^{2}+\widetilde{e}_{1}^{2}+\widetilde{e}_{2}^{2}+\widetilde{e}_{3}^{2}+\widetilde{d}_{1}^{2}\right\}
\end{aligned}
$$

then $\dot{V}<0$ if the adaptive laws for $\widetilde{a}_{o}, \widehat{a}_{o}, \widetilde{b}_{o}, \widehat{b}_{o}, \widetilde{c}_{o}, \widehat{c}_{o}, \widetilde{e}_{r o}, \widehat{e}_{r o}$, $\widetilde{d}_{1}, \widehat{d}_{1}, o=1,2,3$ and the values of $v_{s o}, o=1,2,3$ are chosen as

$$
\begin{aligned}
& \dot{z}_{1}=-e_{r 21}-v_{01}, \\
& v_{s 1}=-k_{1} s_{1} \\
& \dot{z}_{2}=-e_{r 22}-v_{02}, \\
& v_{s 2}=-k_{2} s_{2} \\
& \dot{z}_{3}=-e_{r 23}-v_{03}, \\
& v_{s 3}=-k_{3} s_{3} \\
& \dot{\tilde{a}}_{1}=-\sigma_{1} x_{11}-k_{4} \tilde{a}_{1}, \\
& \dot{\hat{a}}_{1}=-\dot{\tilde{a}}_{1} \\
& \dot{\tilde{a}}_{2}=-2 \sigma_{1} x_{21}-k_{5} \tilde{a}_{2}, \\
& \dot{\vec{a}}_{2}=-\dot{\tilde{a}}_{2} \\
& \dot{\tilde{a}}_{3}=-\sigma_{1} x_{31}-k_{6} \tilde{a}_{3},
\end{aligned}
$$

$$
\begin{aligned}
& \dot{\hat{a}}_{3}=-\dot{\tilde{a}}_{3} \\
& \dot{\widetilde{b}}_{1}=-\sigma_{1} x_{12}-k_{7} \tilde{b}_{1} \text {, } \\
& \dot{\hat{b}}_{1}=-\dot{\widetilde{b}}_{1} \\
& \dot{\tilde{b}}_{2}=-2 \sigma_{1} x_{22}-k_{8} \tilde{b}_{2} \text {, } \\
& \dot{\vec{b}}_{2}=-\dot{\widetilde{b}}_{2} \\
& \dot{\tilde{b}}_{3}=-\sigma_{1} x_{32}-k_{9} \widetilde{b}_{3}, \\
& \dot{\vec{b}}_{3}=-\dot{\widetilde{b}}_{3} \\
& \dot{\tilde{c}}_{1}=-\sigma_{2} x_{11}-k_{10} \widetilde{c}_{1} \text {, } \\
& \dot{\widehat{c}}_{1}=-\dot{\widetilde{c}}_{1} \\
& \dot{\tilde{c}}_{2}=-2 \sigma_{2} x_{22}-k_{11} \widetilde{c}_{2} \text {, } \\
& \dot{\widehat{c}}_{2}=-\dot{\tilde{c}}_{2} \\
& \dot{\tilde{c}}_{3}=-\sigma_{2} x_{31}-k_{12} \widetilde{c}_{3} \text {, } \\
& \dot{\widehat{c}}_{3}=-\dot{\widetilde{c}}_{3} \\
& \dot{\tilde{e}}_{r 1}=-\sigma_{3} x_{13}-k_{13} \widetilde{e}_{r 1} \text {, } \\
& \dot{\hat{e}}_{r 1}=-\dot{\widetilde{e}}_{r 1} \\
& \dot{\tilde{e}}_{r 2}=-2 \sigma_{3} x_{23}-k_{14} \widetilde{e}_{r 2} \text {, } \\
& \dot{\hat{e}}_{r 2}=-\dot{\tilde{e}}_{r 2} \\
& \dot{\tilde{e}}_{r 3}=-\sigma_{3} x_{33}-k_{15} \widetilde{e}_{r 3} \text {, } \\
& \dot{\hat{e}}_{r 3}=-\dot{\tilde{e}}_{r 3} \\
& \dot{\tilde{d}}_{1}=-\sigma_{2} x_{12}-k_{16} \widetilde{d}_{1} \text {, } \\
& \dot{\vec{d}}_{1}=-\dot{\vec{d}}_{1} \text {, }
\end{aligned}
$$$$
k_{o}>0, o=1,2, \ldots, 16
$$

We have

$$
\begin{aligned}
\dot{V}= & -k_{2} \sigma_{1}^{2}-k_{2} \sigma_{2}^{2}-k_{3} \sigma_{3}^{2}-k_{4} \widetilde{a}_{1}^{2}-k_{5} \widetilde{a}_{2}^{2}-k_{6} \widetilde{a}_{3}^{2} \\
& -k_{7} \widetilde{b}_{1}^{2}-k_{8} \widetilde{b}_{2}^{2}-k_{9} \widetilde{b}_{3}^{2}-k_{10} \widetilde{c}_{1}^{2}-k_{11} \widetilde{c}_{2}^{2}-k_{12} \widetilde{c}_{3}^{2} \\
& -k_{13} \widetilde{e}_{r 1}^{2}-k_{14} \widetilde{e}_{r 2}^{2}-k_{15} \widetilde{e}_{r 3}^{2}-k_{16} \widetilde{d}_{1}^{2} .
\end{aligned}
$$

From this we conclude that $\sigma_{o}, \widetilde{e}_{r o}, \widetilde{d}_{1}, \widetilde{a}_{o}, \widetilde{b}_{o}, \widetilde{c}_{o} \rightarrow 0$, o = $1,2,3$. Since $\sigma_{o} \rightarrow 0, o=1,2,3$, therefore $e_{r 1}, e_{r 2} \rightarrow$ 0 . Therefore, antisynchronization is realized. For complete synchronization, the error is $e_{r 1}^{\prime}=x_{3}-x_{1}$, which can be written as $e_{r 1}^{\prime}=x_{3}-x_{1}=x_{3}+x_{2}-x_{2}-x_{1}=e_{r 2}-e_{r 1}$. As 
$e_{r 1}, e_{r 2} \rightarrow 0$ therefore $e_{r 1}^{\prime} \rightarrow 0$. Thus complete synchronization is achieved. Results of simulations are depicted in Figures $2-4$.

Example $2(M=4)$. In this example, only Lorenz systems with unknown parameters are chosen to examine the effectiveness of the proposed method.

These can be expressed as

$$
\begin{aligned}
& \dot{x}_{11}=-10 x_{11}+10 x_{12}+d_{11}^{\prime}\left(x_{41}-x_{11}\right) \\
& \dot{x}_{12}=28 x_{11}-x_{12}-x_{11} x_{13}+d_{12}^{\prime}\left(x_{42}-x_{12}\right) \\
& \dot{x}_{13}=-\frac{8}{3} x_{13}+x_{11} x_{12}+d_{13}^{\prime}\left(x_{43}-x_{13}\right) \\
& \dot{x}_{21}=-10 x_{21}+10 x_{22}+d_{21}^{\prime}\left(x_{11}-x_{21}\right)+u_{11}^{\prime} \\
& \dot{x}_{22}=28 x_{21}-x_{22}-x_{21} x_{23}+d_{22}^{\prime}\left(x_{12}-x_{22}\right)+u_{12}^{\prime} \\
& \dot{x}_{23}=-\frac{8}{3} x_{23}+x_{21} x_{22}+d_{23}^{\prime}\left(x_{13}-x_{23}\right)+u_{13}^{\prime} \\
& \dot{x}_{31}=-10 x_{31}+10 x_{32}+d_{31}^{\prime}\left(x_{21}-x_{31}\right)+u_{21}^{\prime} \\
& \dot{x}_{32}=28 x_{31}-x_{32}-x_{31} x_{33}+d_{32}^{\prime}\left(x_{22}-x_{32}\right)+u_{22}^{\prime} \\
& \dot{x}_{33}=-\frac{8}{3} x_{33}+x_{31} x_{32}+d_{33}^{\prime}\left(x_{23}-x_{33}\right)+u_{23}^{\prime} \\
& \dot{x}_{41}=-10 x_{41}+10 x_{42}+d_{41}^{\prime}\left(x_{31}-x_{41}\right)+u_{31}^{\prime} \\
& \dot{x}_{42}=28 x_{41}-x_{42}-x_{41} x_{43}+d_{42}^{\prime}\left(x_{32}-x_{42}\right)+u_{32}^{\prime} \\
& \dot{x}_{43}=-\frac{8}{3} x_{43}+x_{41} x_{42}+d_{43}^{\prime}\left(x_{33}-x_{43}\right)+u_{33}^{\prime} .
\end{aligned}
$$

With unknown parameters above four systems can be represented as

$$
\begin{aligned}
& \dot{x}_{11}=a x_{11}+b x_{12}+d_{11}^{\prime}\left(x_{41}-x_{11}\right) \\
& \dot{x}_{12}=c x_{11}-x_{12}-x_{11} x_{13}+d_{12}^{\prime}\left(x_{42}-x_{12}\right) \\
& \dot{x}_{13}=d x_{13}+x_{11} x_{12}+d_{13}^{\prime}\left(x_{43}-x_{13}\right) \\
& \dot{x}_{21}=a x_{21}+b x_{22}+d_{21}^{\prime}\left(x_{11}-x_{21}\right)+u_{11}^{\prime} \\
& \dot{x}_{22}=c x_{21}-x_{22}-x_{21} x_{23}+d_{22}^{\prime}\left(x_{12}-x_{22}\right)+u_{12}^{\prime} \\
& \dot{x}_{23}=d x_{23}+x_{21} x_{22}+d_{23}^{\prime}\left(x_{13}-x_{23}\right)+u_{13}^{\prime} \\
& \dot{x}_{31}=a x_{31}+b x_{32}+d_{31}^{\prime}\left(x_{21}-x_{31}\right)+u_{21}^{\prime} \\
& \dot{x}_{32}=c x_{31}-x_{32}-x_{31} x_{33}+d_{32}^{\prime}\left(x_{22}-x_{32}\right)+u_{22}^{\prime} \\
& \dot{x}_{33}=d x_{33}+x_{31} x_{32}+d_{33}^{\prime}\left(x_{23}-x_{33}\right)+u_{23}^{\prime} \\
& \dot{x}_{41}=a x_{41}+b x_{42}+d_{41}^{\prime}\left(x_{31}-x_{41}\right)+u_{31}^{\prime} \\
& \dot{x}_{42}=c x_{41}-x_{42}-x_{41} x_{43}+d_{42}^{\prime}\left(x_{32}-x_{42}\right)+u_{32}^{\prime} \\
& \dot{x}_{43}=d x_{43}+x_{41} x_{42}+d_{43}^{\prime}\left(x_{33}-x_{43}\right)+u_{33}^{\prime} .
\end{aligned}
$$

Let $\widehat{a}, \widehat{b}, \widehat{c}, \widehat{d}$ be estimates of $a, b, c, d$, respectively, and let $\widetilde{a}=$ $a-\widehat{a}, \widetilde{b}_{i}=b-\widehat{b}, \widetilde{c}=c-\widehat{c}, \widetilde{d}_{1}=d-\widehat{d}$ be the errors in estimation of $a, b, c, d$, respectively.

Defining

$$
\begin{aligned}
& x_{1}=\left[\begin{array}{l}
x_{11} \\
x_{12} \\
x_{13}
\end{array}\right], \\
& x_{2}=\left[\begin{array}{l}
x_{21} \\
x_{22} \\
x_{23}
\end{array}\right] \text {, } \\
& x_{3}=\left[\begin{array}{l}
x_{31} \\
x_{32} \\
x_{33}
\end{array}\right] \text {, } \\
& x_{4}=\left[\begin{array}{l}
x_{41} \\
x_{42} \\
x_{43}
\end{array}\right] \text {, } \\
& u_{1}^{\prime}=\left[\begin{array}{l}
u_{11}^{\prime} \\
u_{12}^{\prime} \\
u_{13}^{\prime}
\end{array}\right] \text {, } \\
& u_{2}^{\prime}=\left[\begin{array}{l}
u_{21}^{\prime} \\
u_{22}^{\prime} \\
u_{23}^{\prime}
\end{array}\right] \text {, } \\
& u_{3}^{\prime}=\left[\begin{array}{l}
u_{31}^{\prime} \\
u_{32}^{\prime} \\
u_{33}^{\prime}
\end{array}\right] \text {, } \\
& \widehat{\theta}=\left[\begin{array}{l}
\widehat{a} \\
\widehat{b} \\
\widehat{c} \\
\widehat{d}
\end{array}\right] \\
& \widetilde{\theta}=\left[\begin{array}{c}
\widetilde{a} \\
\widetilde{b} \\
\widetilde{c} \\
\widetilde{d}
\end{array}\right]
\end{aligned}
$$

systems (43)-(45) are represented as

$$
\begin{aligned}
& \dot{x}_{1}=f_{1}\left(x_{1}\right)+F_{1}\left(x_{1}\right) \hat{\theta}+F_{1}\left(x_{1}\right) \tilde{\theta} \\
& \dot{x}_{2}=f_{2}\left(x_{2}\right)+F_{2}\left(x_{2}\right) \hat{\theta}+F_{2}\left(x_{2}\right) \tilde{\theta}+u_{1}^{\prime} \\
& \dot{x}_{3}=f_{3}\left(x_{3}\right)+F_{3}\left(x_{3}\right) \hat{\theta}+F_{3}\left(x_{3}\right) \tilde{\theta}+u_{2}^{\prime} \\
& \dot{x}_{4}=f_{4}\left(x_{4}\right)+F_{4}\left(x_{4}\right) \hat{\theta}+F_{4}\left(x_{4}\right) \tilde{\theta}+u_{3}^{\prime},
\end{aligned}
$$


where

$$
\begin{aligned}
& f_{1}\left(x_{1}\right)=\left[\begin{array}{c}
d_{11}^{\prime}\left(x_{41}-x_{11}\right) \\
-x_{12}-x_{11} x_{13}+d_{12}^{\prime}\left(x_{42}-x_{12}\right) \\
x_{11} x_{12}+d_{13}^{\prime}\left(x_{43}-x_{13}\right)
\end{array}\right], \\
& F_{1}\left(x_{1}\right)=\left[\begin{array}{cccc}
x_{11} & x_{12} & 0 & 0 \\
0 & 0 & x_{11} & 0 \\
0 & 0 & 0 & x_{13}
\end{array}\right] \\
& f_{2}\left(x_{2}\right)=\left[\begin{array}{c}
d_{21}^{\prime}\left(x_{11}-x_{21}\right) \\
-x_{22}-x_{21} x_{23}+d_{22}^{\prime}\left(x_{12}-x_{22}\right) \\
x_{21} x_{22}+d_{23}^{\prime}\left(x_{13}-x_{23}\right)
\end{array}\right] \\
& F_{2}\left(x_{2}\right)=\left[\begin{array}{cccc}
x_{21} & x_{22} & 0 & 0 \\
0 & 0 & x_{21} & 0 \\
0 & 0 & 0 & x_{23}
\end{array}\right] \\
& f_{3}\left(x_{3}\right)=\left[\begin{array}{c}
d_{31}^{\prime}\left(x_{21}-x_{31}\right) \\
-x_{32}-x_{31} x_{33}+d_{32}^{\prime}\left(x_{22}-x_{32}\right) \\
x_{31} x_{32}+d_{33}^{\prime}\left(x_{23}-x_{33}\right)
\end{array}\right], \\
& F_{3}\left(x_{3}\right)=\left[\begin{array}{cccc}
x_{31} & x_{32} & 0 & 0 \\
0 & 0 & x_{31} & 0 \\
0 & 0 & 0 & x_{33}
\end{array}\right] \\
& f_{4}\left(x_{4}\right)=\left[\begin{array}{c}
d_{41}^{\prime}\left(x_{31}-x_{41}\right) \\
-x_{42}-x_{41} x_{43}+d_{42}^{\prime}\left(x_{32}-x_{42}\right) \\
x_{41} x_{42}+d_{43}^{\prime}\left(x_{33}-x_{43}\right)
\end{array}\right] \\
& F_{4}\left(x_{4}\right)=\left[\begin{array}{cccc}
x_{41} & x_{42} & 0 & 0 \\
0 & 0 & x_{41} & 0 \\
0 & 0 & 0 & x_{43}
\end{array}\right]
\end{aligned}
$$

For the antisynchronization, the error vectors

$$
\begin{aligned}
& e_{r 1}=\left[\begin{array}{l}
e_{r 11} \\
e_{r 12} \\
e_{r 13}
\end{array}\right], \\
& e_{r 2}=\left[\begin{array}{l}
e_{r 21} \\
e_{r 22} \\
e_{r 23}
\end{array}\right], \\
& e_{r 3}=\left[\begin{array}{l}
e_{r 31} \\
e_{r 32} \\
e_{r 33}
\end{array}\right]
\end{aligned}
$$

are

$$
\begin{aligned}
& e_{r 1}=x_{2}+x_{1}, \\
& e_{r 2}=x_{3}+x_{2}, \\
& e_{r 3}=x_{4}+x_{3} .
\end{aligned}
$$

Therefore

$$
\begin{aligned}
{\left[\begin{array}{c}
\dot{e}_{r 1} \\
\dot{e}_{r 2} \\
\dot{e}_{r 3}
\end{array}\right]=} & {\left[\begin{array}{l}
\left(F_{1}\left(x_{1}\right)+F_{2}\left(x_{2}\right)\right) \hat{\theta}+f_{1}\left(x_{1}\right)+f_{2}\left(x_{2}\right) \\
\left(F_{2}\left(x_{2}\right)+F_{3}\left(x_{3}\right)\right) \hat{\theta}+f_{2}\left(x_{2}\right)+f_{3}\left(x_{3}\right) \\
\left(F_{3}\left(x_{3}\right)+F_{4}\left(x_{4}\right)\right) \hat{\theta}+f_{3}\left(x_{3}\right)+f_{4}\left(x_{4}\right)
\end{array}\right] } \\
+ & {\left[\begin{array}{l}
F_{1}\left(x_{1}\right)+F_{2}\left(x_{2}\right) \\
F_{2}\left(x_{2}\right)+F_{3}\left(x_{3}\right) \\
F_{3}\left(x_{3}\right)+F_{4}\left(x_{4}\right)
\end{array}\right] \tilde{\theta} } \\
& +\left[\begin{array}{lll}
1 & 0 & 0 \\
1 & 1 & 0 \\
0 & 1 & 1
\end{array}\right]\left[\begin{array}{l}
u_{1}^{\prime} \\
u_{2}^{\prime} \\
u_{3}^{\prime}
\end{array}\right] .
\end{aligned}
$$

\section{Choose}

$$
\begin{aligned}
& {\left[\begin{array}{l}
u_{1}^{\prime} \\
u_{2}^{\prime} \\
u_{3}^{\prime}
\end{array}\right]=\left[\begin{array}{lll}
1 & 0 & 0 \\
1 & 1 & 0 \\
0 & 1 & 1
\end{array}\right]^{-1}} \\
& \cdot\left\{-\left[\begin{array}{l}
f_{2}\left(x_{2}\right)+f_{1}\left(x_{1}\right)+\left(F_{2}\left(x_{2}\right)+F_{1}\left(x_{1}\right)\right) \hat{\theta} \\
f_{3}\left(x_{3}\right)+f_{2}\left(x_{2}\right)+\left(F_{3}\left(x_{3}\right)+F_{2}\left(x_{2}\right)\right) \hat{\theta} \\
f_{4}\left(x_{4}\right)+f_{3}\left(x_{3}\right)+\left(F_{4}\left(x_{4}\right)+F_{3}\left(x_{3}\right)\right) \hat{\theta}
\end{array}\right]\right. \\
& \left.+\left[\begin{array}{c}
e_{r 2} \\
e_{r 3} \\
v
\end{array}\right]\right\}
\end{aligned}
$$

where $v$ is the new input vector.

We have

$$
\left[\begin{array}{c}
\dot{e}_{r 1} \\
\dot{e}_{r 2} \\
\dot{e}_{r 3}
\end{array}\right]=\left[\begin{array}{c}
e_{r 2} \\
e_{r 3} \\
v
\end{array}\right]+\left[\begin{array}{c}
F_{2}\left(x_{2}\right)+F_{1}\left(x_{1}\right) \\
F_{3}\left(x_{3}\right)+F_{2}\left(x_{2}\right) \\
F_{4}\left(x_{4}\right)+F_{3}\left(x_{3}\right)
\end{array}\right] \tilde{\theta}
$$

or

$$
\begin{aligned}
& \dot{e}_{r 1}=e_{r 2}+\left(F_{2}\left(x_{2}\right)+F_{1}\left(x_{1}\right)\right) \tilde{\theta} \\
& \dot{e}_{r 2}=e_{r 3}+\left(F_{3}\left(x_{3}\right)+F_{2}\left(x_{2}\right)\right) \tilde{\theta} \\
& \dot{e}_{r 3}=v+\left(F_{4}\left(x_{4}\right)+F_{3}\left(x_{3}\right)\right) \tilde{\theta} .
\end{aligned}
$$

The nominal system for (54) is

$$
\begin{aligned}
& \dot{e}_{r 1}=e_{r 2} \\
& \dot{e}_{r 2}=e_{r 3} \\
& \dot{e}_{r 3}=v_{0} .
\end{aligned}
$$


The Hurwitz sliding surface vector for nominal system (55) can be defined as

$$
\sigma_{0}=e_{r 1}+2 e_{r 2}+e_{r 3}
$$

where

$$
\sigma_{0}=\left[\begin{array}{l}
\sigma_{01} \\
\sigma_{02} \\
\sigma_{03}
\end{array}\right]=\left[\begin{array}{l}
e_{r 11}+2 e_{r 21}+e_{r 31} \\
e_{r 12}+2 e_{r 22}+e_{r 32} \\
e_{r 13}+2 e_{r 23}+e_{r 33}
\end{array}\right] \text {. }
$$

Then the derivative of the above system is $\dot{\sigma}_{0}=\dot{e}_{r 1}+2 \dot{e}_{r 2}=$ $e_{r 2}+2 e_{r 3}+v_{0}$. By choosing $v_{0}=-e_{r 2}-2 e_{r 3}-k \sigma_{0}-$ $k \operatorname{sign}\left(\sigma_{0}\right), k>0$, we have $\dot{\sigma}_{0}=-k \operatorname{sign}\left(\sigma_{0}\right)-k \sigma_{0}$. So the error dynamics (55) are asymptotically stable.

Sliding surface for the system (55) is described as $\sigma=\sigma_{0}+$ $z=e_{r 1}+2 e_{r 2}+e_{r 3}+z$, where $z=\left[\begin{array}{lll}z_{1} & z_{3} & z_{3}\end{array}\right]^{T}$ is some integral term, by choosing $z(0)$ in such a way that $\sigma(0)=0$. Select $v=v_{0}+v_{s}$, where $v_{0}=\left[\begin{array}{lll}v_{01} & v_{02} & v_{03}\end{array}\right]^{T}$ is the nominal input and $v_{s}=\left[\begin{array}{lll}v_{s 1} & v_{s 2} & v_{s 3}\end{array}\right]^{T}$ is compensator term computed later.

The system (54) can be rewritten as

$$
\begin{aligned}
& \dot{e}_{r 11}=e_{r 21}+\widetilde{a} x_{21}+\widetilde{b} x_{22}+\widetilde{a} x_{11}+\widetilde{b} x_{12} \\
& \dot{e}_{r 12}=e_{r 22}+\widetilde{c} x_{21}+\widetilde{c} x_{11} \\
& \dot{e}_{r 13}=e_{r 23}+\tilde{d} x_{23}+\tilde{d} x_{13} \\
& \dot{e}_{r 21}=e_{r 31}+\tilde{a} x_{31}+\tilde{b} x_{32}+\tilde{a} x_{21}+\tilde{b} x_{22} \\
& \dot{e}_{r 22}=e_{r 32}+\tilde{c} x_{31}+\tilde{c} x_{21} \\
& \dot{e}_{r 23}=e_{r 33}+\tilde{d} x_{33}+\tilde{d} x_{23} \\
& \dot{e}_{r 31}=v_{01}+v_{s 1}+\widetilde{a} x_{41}+\widetilde{b} x_{42}+\widetilde{a} x_{31}+\widetilde{b} x_{32} \\
& \dot{e}_{r 32}=v_{02}+v_{s 2}+\tilde{c} x_{41}+\widetilde{c} x_{31} \\
& \dot{e}_{r 33}=v_{03}+v_{s 3}+\tilde{d} x_{43}+\tilde{d} x_{33} \text {. }
\end{aligned}
$$

Then $\dot{\sigma}=\dot{e}_{r 1}+2 \dot{e}_{r 2}+\dot{e}_{r 3}+\dot{z}$ gives

$$
\begin{aligned}
\dot{\sigma}_{1}= & \dot{e}_{r 11}+2 \dot{e}_{r 21}+\dot{e}_{r 31}+\dot{z}_{1} \\
= & e_{r 21}+\widetilde{a} x_{21}+\widetilde{b} x_{22}+\widetilde{a} x_{11}+\widetilde{b} x_{12}+2 e_{r 31} \\
& +2 \widetilde{a} x_{31}+2 \widetilde{b} x_{32}+2 \widetilde{a} x_{21}+2 \widetilde{b} x_{22}+v_{01}+v_{s 1} \\
& +\widetilde{a} x_{41}+\tilde{b} x_{42}+\tilde{a} x_{31}+\widetilde{b} x_{32}+\dot{z}_{1} \\
\dot{\sigma}_{2}= & \dot{e}_{r 12}+2 \dot{e}_{r 22}+\dot{e}_{r 32}+\dot{z}_{2} \\
= & e_{r 22}+\widetilde{c} x_{21}+q \widetilde{c} x_{11}+2 e_{r 32}+2 \widetilde{c} x_{31}+2 \widetilde{c} x_{21} \\
& +v_{02}+v_{s 2}+\widetilde{c} x_{41}+\widetilde{c} x_{31}+\dot{z} 2 \\
\dot{\sigma}_{3}= & \dot{e}_{r 13}+2 \dot{e}_{r 23}+\dot{e}_{r 33}+\dot{z}_{3} \\
= & e_{r 23}+\widetilde{d} x_{23}+\widetilde{d} x_{13}+2 e_{r 33}+2 \widetilde{d} x_{33}+2 \widetilde{d} x_{23} \\
& +v_{03}+v_{s 3}+\tilde{d} x_{43}+\tilde{d} x_{33}+\dot{z}_{3}
\end{aligned}
$$

or

$$
\begin{aligned}
\dot{\sigma}_{1}= & e_{r 21}+2 e_{r 31}+\tilde{a}\left\{x_{11}+3 x_{21}+3 x_{31}+x_{41}\right\} \\
& +\widetilde{b}\left\{x_{12}+3 x_{22}+3 x_{32}+x_{42}\right\}+v_{01}+v_{s 1} \\
& +\dot{z}_{1} \\
\dot{\sigma}_{2}= & e_{r 22}+2 e_{r 32}+\widetilde{c}\left\{x_{11}+3 x_{21}+3 x_{31}+x_{41}\right\}+v_{02} \\
& +v_{s 2}+\dot{z}_{2} \\
\dot{\sigma}_{3}= & e_{r 23}+2 e_{r 33}+\tilde{d}\left\{x_{13}+3 x_{23}+3 x_{33}+x_{43}\right\}+v_{03} \\
& +v_{s 3}+\dot{z}_{3} .
\end{aligned}
$$

Choose the Lyapunov function: $V=(1 / 2)\left\{\sigma_{1}^{2}+\sigma_{2}^{2}+\sigma_{3}^{2}+\widetilde{a}^{2}+\right.$ $\left.\widetilde{b}^{2}+\widetilde{c}^{2}+\widetilde{d}^{2}\right\}$.

Then $\dot{V}<0$ if the adaptive laws for $\widetilde{a}, \widetilde{b}, \widetilde{c}, \widetilde{d}, \widehat{a}, \widehat{b}, \widehat{c}, \widehat{d}$ and $v_{s o}, o=1,2,3$ are chosen as

$$
\begin{aligned}
& \dot{z}_{1}=-e_{r 21}-2 e_{r 31}-v_{01}, \\
& v_{s 1}=-k_{1} s_{1} \\
& \dot{z}_{2}=-e_{r 22}-2 e_{r 32}-v_{02}, \\
& v_{s 2}=-k_{2} s_{2} \\
& \dot{z}_{3}=-e_{r 23}-2 e_{r 33}-v_{03}, \\
& v_{s 3}=-k_{3} s_{3} \\
& \dot{\tilde{a}}=-\sigma_{1}\left(x_{11}+3 x_{21}+3 x_{31}+x_{41}\right)-k_{4} \tilde{a}, \\
& \dot{\hat{a}}=-\dot{\tilde{a}} \\
& \dot{\tilde{b}}=-\sigma_{1}\left(x_{12}+3 x_{22}+3 x_{32}+x_{42}\right)-k_{5} \tilde{b}, \\
& \dot{\hat{b}}=-\dot{\widetilde{b}} \\
& \dot{\tilde{c}}=-\sigma_{2}\left(x_{11}+3 x_{21}+3 x_{31}+x_{41}\right)-k_{6} \tilde{c}, \\
& \dot{\hat{c}}=-\dot{\tilde{c}} \\
& \dot{\tilde{d}}=-\sigma_{3}\left(x_{13}+3 x_{23}+3 x_{33}+x_{43}\right)-k_{7} \tilde{d}, \\
& \dot{\hat{d}}=-\dot{\tilde{d}},
\end{aligned}
$$

From this we conclude that $\widetilde{a}, \widetilde{b}, \widetilde{c}, \widetilde{e}_{r}, \sigma_{1}, \sigma_{2}, \sigma_{3}, \widetilde{d} \rightarrow 0$. Since $\sigma_{o} \rightarrow 0, o=1,2,3$, therefore $e_{r 1}, e_{r 2}, e_{r 3} \rightarrow 0$. Thus antisynchronization is achieved.

For complete synchronization, the errors are $e_{r 1}^{\prime}=x_{3}-$ $x_{1}, e_{r 2}^{\prime}=x_{4}-x_{2}$ which can be written as

$$
\begin{aligned}
& e_{r 1}^{\prime}=x_{3}-x_{1}=x_{3}+x_{2}-x_{2}-x_{1}=e_{r 2}-e_{r 1} \\
& e_{r 2}^{\prime}=x_{4}-x_{2}=x_{4}+x_{3}-x_{3}-x_{2}=e_{r 3}-e_{r 2} .
\end{aligned}
$$

As $e_{r 1}, e_{r 2}, e_{r 3} \rightarrow 0$ therefore $e_{r 1}^{\prime}, e_{r 2}^{\prime} \rightarrow 0$; thus complete synchronization is achieved. Simulation results are shown in Figures 5-7. 


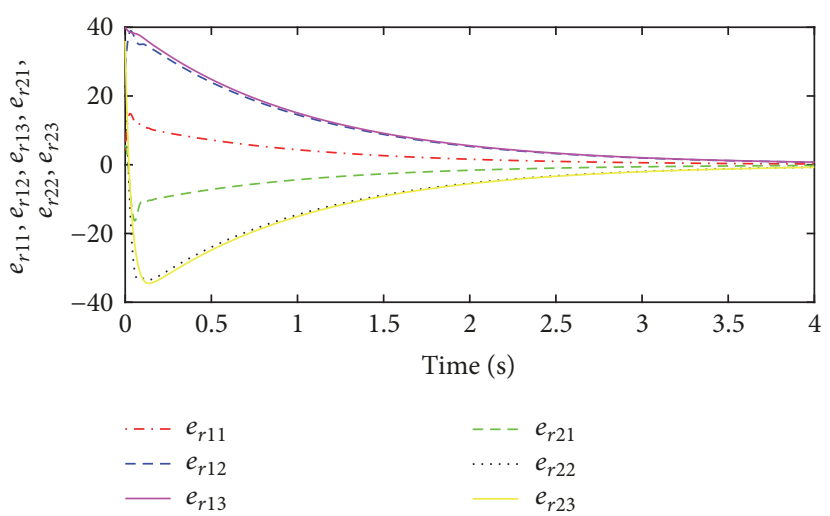

(a) Convergence of error system $e_{r 11}, e_{r 12}, e_{r 13}, e_{r 21}, e_{r 22}, e_{r 23}$

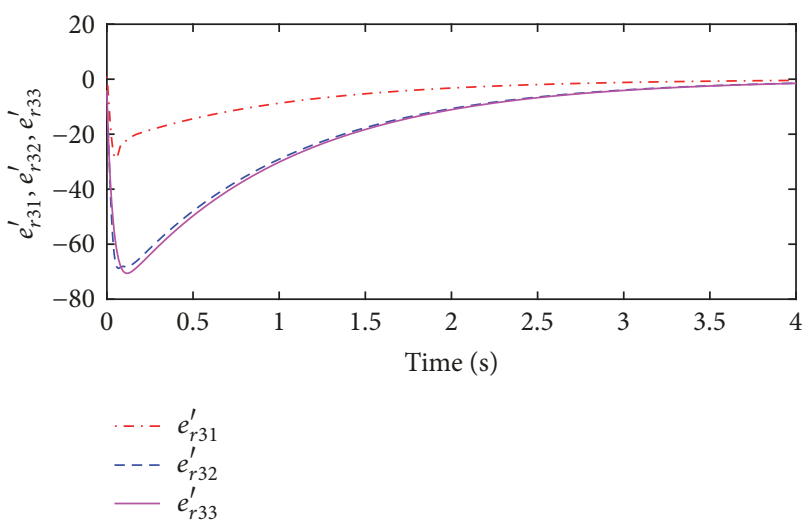

(b) Convergence of error system $e_{r 31}^{\prime}, e_{r 32}^{\prime}, e_{r 33}^{\prime}$

FiguRe 2

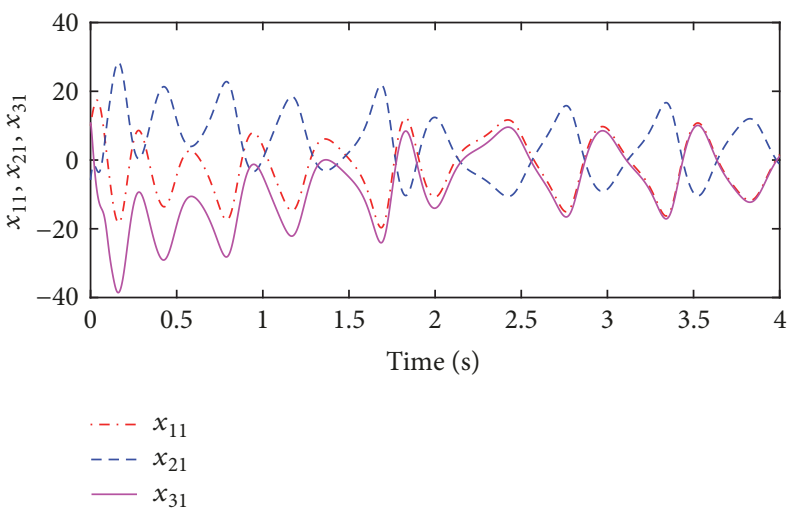

(a) Time history of system states $x_{11}, x_{21}$, and $x_{31}$

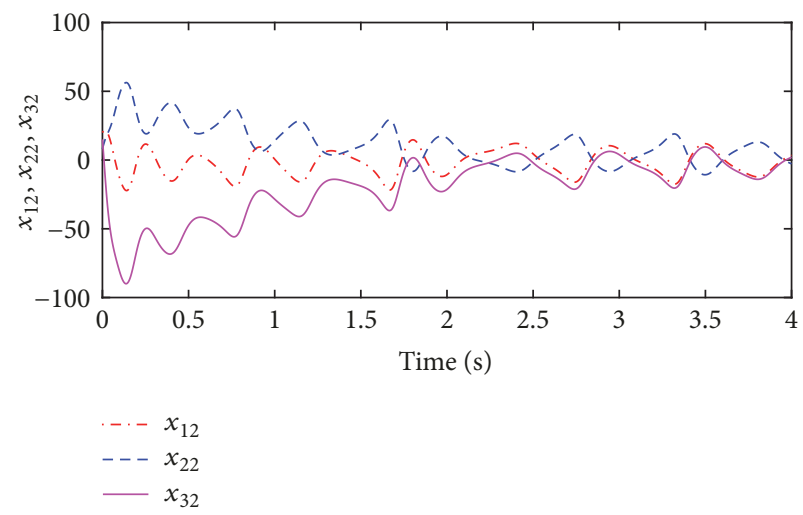

(b) Time history of system states $x_{12}, x_{22}$, and $x_{32}$

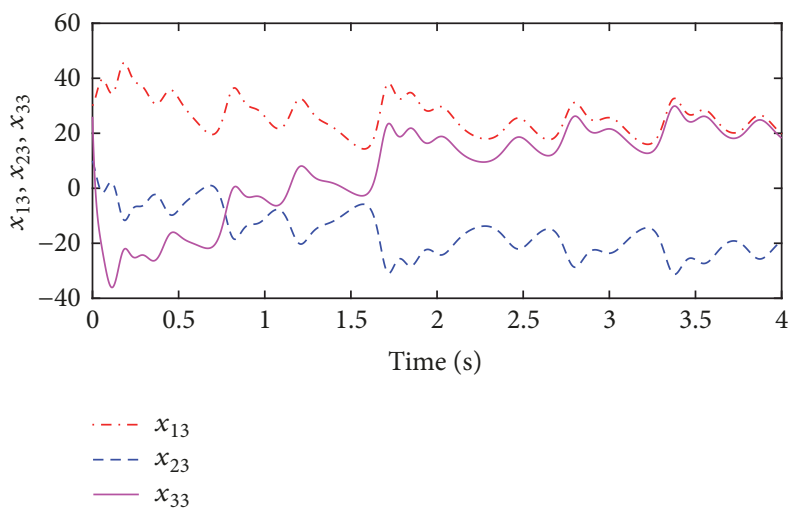

(c) Time history of system states $x_{13}, x_{23}$, and $x_{33}$

Figure 3

\section{Simulation Results and Discussion}

Figures 2-4 show simulation results for Example 1 with $M=3$. The initial (starting point) conditions are chosen as $\left(x_{11}(0)=10, x_{12}(0)=20, x_{13}(0)=30\right),\left(x_{21}(0)=-5.8\right.$, $\left.x_{22}(0)=8, x_{23}(0)=10\right)$, and $\left(x_{31}(0)=11, x_{32}(0)=15\right.$, $\left.x_{33}(0)=26\right)$. The coupling parameters are chosen as $d_{11}=$ $d_{21}=d_{13}=d_{23}=d_{31}=d_{33}=0, d_{12}=10, d_{22}=11$ and $d_{32}=1$. Figure 2 (a) shows that the errors $e_{r 11}, e_{r 12}$, $e_{r 13}, e_{r 21}, e_{r 22}$, and $e_{r 23}$ asymptotically go to zero. Figure 2(b) displays the errors $e_{r 31}^{\prime}, e_{r 32}^{\prime}$, and $e_{r 33}^{\prime}$, converging to origin. Figure 3(a) shows system states $x_{11}, x_{21}, x_{31}$, Figure 3(b) shows system states $x_{12}, x_{22}$, and $x_{32}$, and Figure 3(c) shows system states $x_{13}, x_{23}$, and $x_{33}$. From these figures we can see that systems $x_{1}(t)$ and $x_{2}(t)$, and systems $x_{2}(t)$ and $x_{3}(t)$ achieve the antisynchronization, and systems $x_{1}(t)$ and $x_{3}(t)$ attain complete synchronization and therefore milestone is attained, that is, hybrid synchronization. Figure 4 shows the 


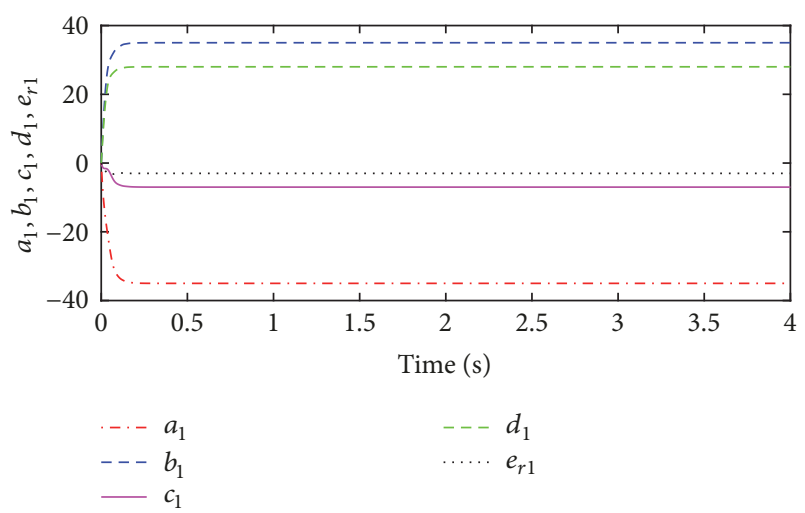

(a) Parameter estimation $a_{1}, b_{1}, c_{1}, d_{1}$, and $e_{r 1}$

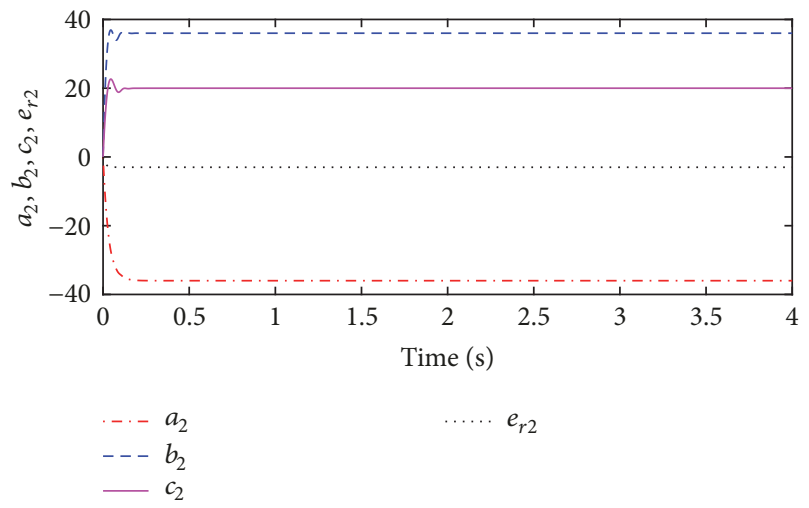

(b) Parameter estimation $a_{2}, b_{2}, c_{2}$, and $e_{r 2}$

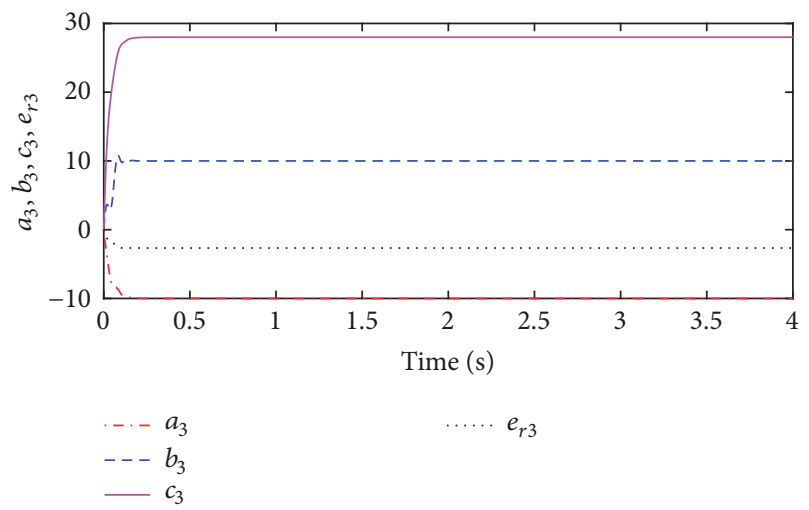

(c) Parameter estimation $a_{3}, b_{3}, c_{3}$, and $e_{r 3}$

FIGURE 4

adaptive estimation of parameters $a, b, c, d$, and $e_{r}$ for the three systems. Figure 4(a) shows the estimation of $a_{1}, b_{1}, c_{1}$, $d_{1}$, and $e_{r 1}$, the parameters of the first system, which converge to their true values of $-35,35,-7,28$, and -3 , respectively. Figure $4(\mathrm{~b})$ shows the estimation of $a_{2}, b_{2}, c_{2}$, and $e_{r 2}$, and the parameters of the second system, which converges to their true values of $-36,36,20$, and 3 , respectively. Figure 4(c) shows the estimation of $a_{3}, b_{3}, c_{3}$, and $e_{r 3}$, the parameters of the third system, which converges to their true values $-10,10$, 28 , and $-8 / 3$, respectively.

Figures 5-7 show simulation results for Example 2 with $M=4$. The initial (starting point) conditions are chosen as

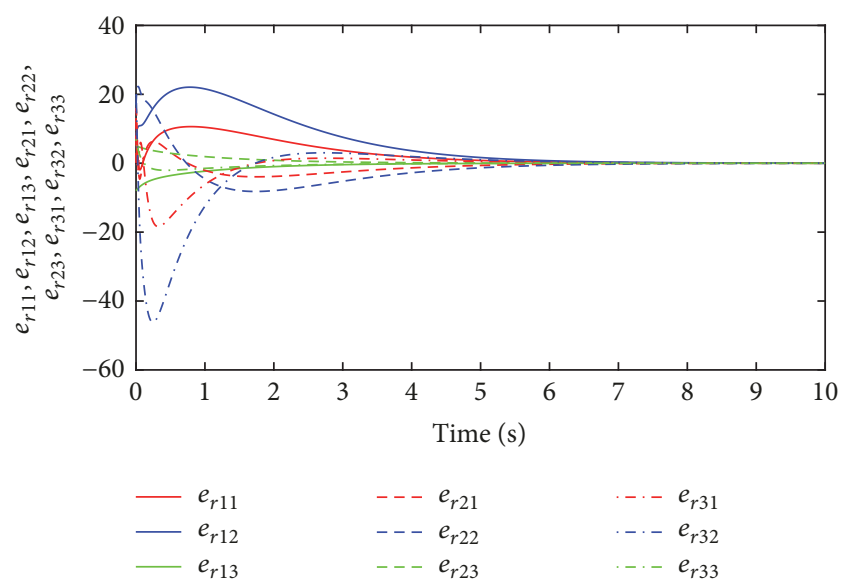

(a) Convergence of error system $e_{r 11}, e_{r 12}, e_{r 13}, e_{r 21}, e_{r 22}, e_{r 23}, e_{r 31}, e_{r 32}$, and $e_{r 33}$

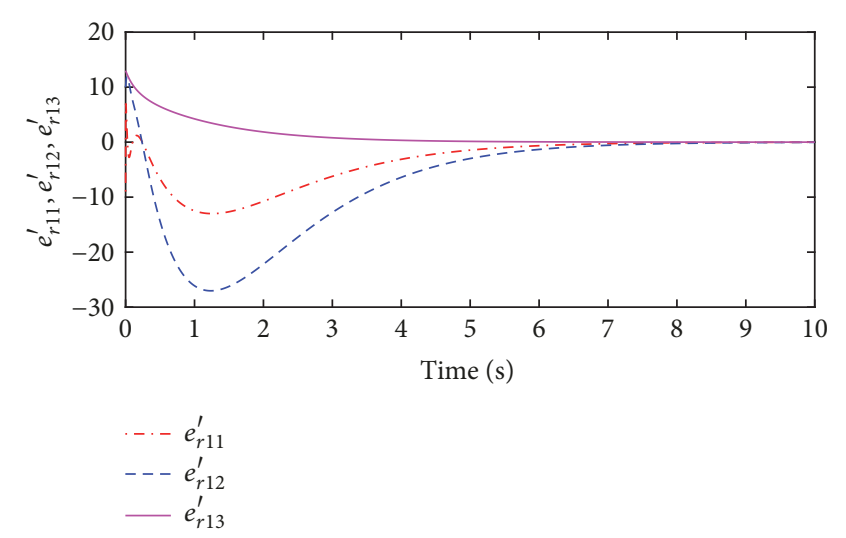

(b) Convergence of error system $e_{r 11}^{\prime}, e_{r 12}^{\prime}$, and $e_{r 13}^{\prime}$

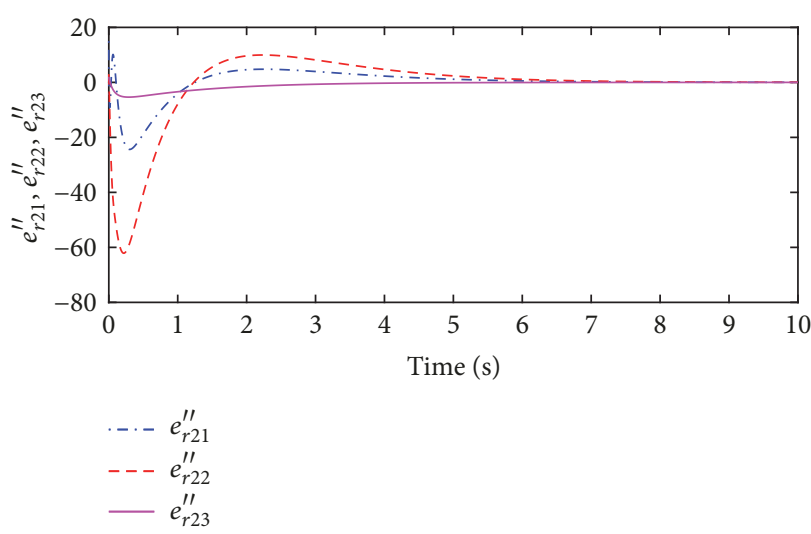

(c) Convergence of error system $e_{r 21}^{\prime \prime}, e_{r 22}^{\prime \prime}$, and $e_{r 23}^{\prime \prime}$

FIGURE 5

$\left(x_{11}(0)=4, x_{12}(0)=5, x_{13}(0)=-3\right),\left(x_{21}(0)=5, x_{22}(0)=2\right.$, $\left.x_{23}(0)=-5\right),\left(x_{31}(0)=11, x_{32}(0)=15, x_{33}(0)=10\right)$, and $\left(x_{41}(0)=4, x_{42}(0)=5, x_{43}(0)=-3\right)$. The coupling parameters are chosen as $d_{12}=d_{22}=d_{32}=d_{13}=d_{23}=$ $d_{33}=d_{42}=d_{43}=0, d_{11}=d_{21}=1$ and $d_{31}=d_{41}=-1$. Figure 5(a) shows the errors $e_{r 11}, e_{r 12}, e_{r 13}, e_{r 21}, e_{r 22}, e_{r 23}$, $e_{r 31}, e_{r 32}$, and $e_{r 33}$ asymptotically converge to zero. Figure 5(b) shows the errors $e_{r 11}^{\prime}, e_{r 12}^{\prime}$, and $e_{r 13}^{\prime}$ asymptotically converge to zero. Figure $5(\mathrm{c})$ shows the errors $e_{r 21}^{\prime \prime}, e_{r 22}^{\prime \prime}$, and $e_{r 23}^{\prime \prime}$ 


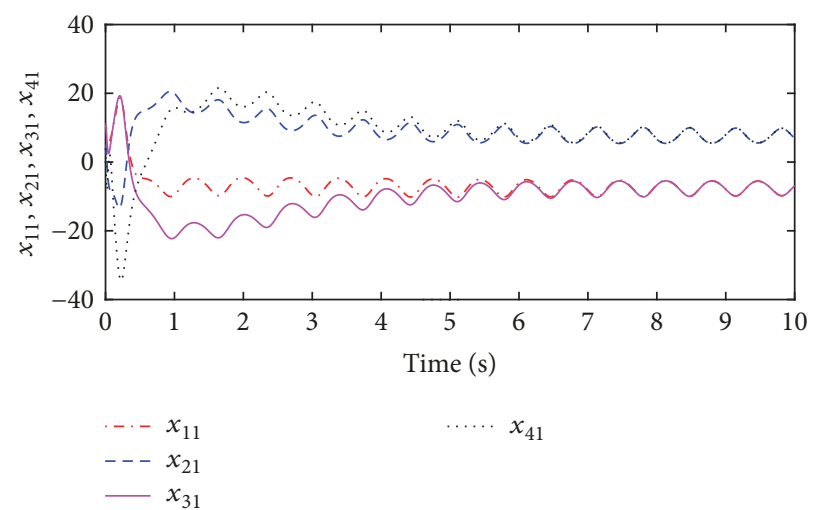

(a) Time history of system states $x_{11}, x_{21}, x_{31}$, and $x_{41}$

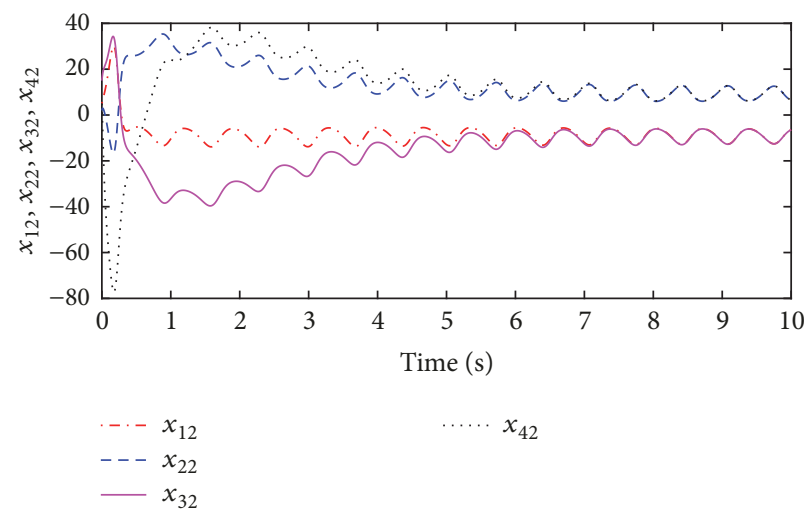

(b) Time history of system states $x_{12}, x_{22}, x_{32}$, and $x_{42}$

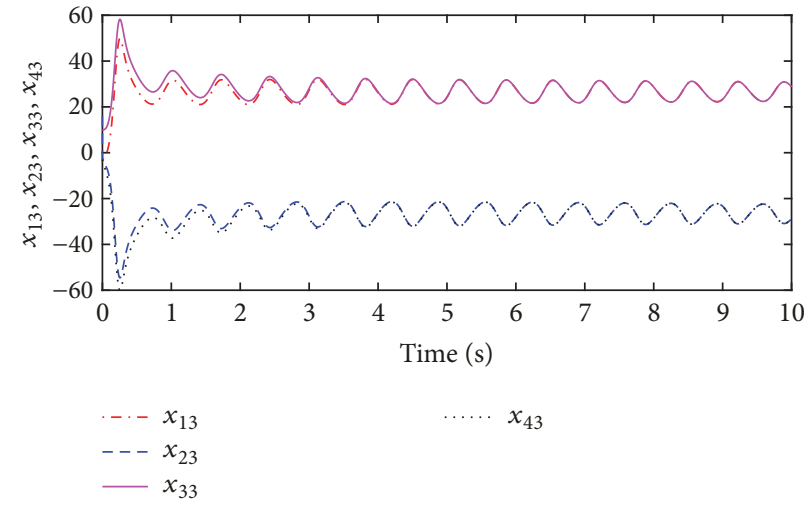

(c) Time history of system states $x_{13}, x_{23}, x_{33}$, and $x_{43}$

Figure 6

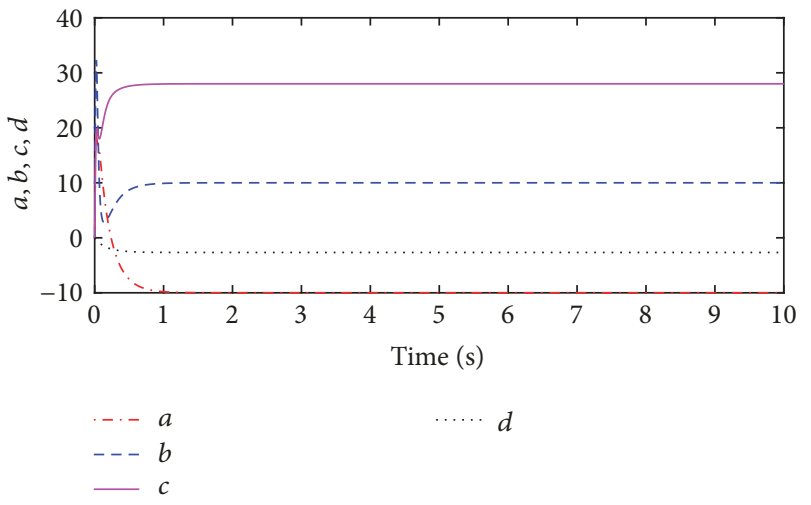

Figure 7: Parameter estimation $a, b, c$, and $d$.

asymptotically converge to zero. Figure 6(a) shows system states $x_{11}, x_{21}, x_{31}$, and $x_{41}$, Figure 6(b) shows system states $x_{12}, x_{22}, x_{32}$, and $x_{42}$, and Figure $6(\mathrm{c})$ shows system states $x_{13}$, $x_{23}, x_{33}$, and $x_{43}$. From these figures we can see that systems $x_{1}(t)$ and $x_{2}(t)$, systems $x_{2}(t)$ and $x_{3}(t)$, and systems $x_{3}(t)$ and $x_{4}(t)$ achieve the antisynchronization, and systems $x_{1}(t)$ and $x_{3}(t)$ and systems $x_{2}(t)$ and $x_{4}(t)$ attained the complete synchronization and hence hybrid synchronization is attained. Figure 7 shows an adaptive estimation of parameters $a, b, c$, and $d$ for four systems which converge to their true values of $-10,10,28$, and $-8 / 3$ respectively.

\section{Conclusion}

In this article, we presented control design method for parameter identification and synchronization/antisynchronization of many attached chaotic systems connected in the ring topology. The methodology is created using adaptive integral SMC. The error system was transformed into a special structure containing nominal part and some unknown terms. The unknown terms were computed adaptively. Then the error system was stabilized using integral sliding mode control. The stabilizing controller for the error system is created that contains the nominal control and the compensator control. Simulation results show that antisynchronization and complete synchronization were achieved with the proposed control laws and that the uncertain parameters converge to their actual values.

\section{Conflicts of Interest}

The authors do not have any conflicts of interest regarding the publication of this paper. 


\section{References}

[1] L. M. Pecora and T. L. Carroll, "Synchronization in chaotic systems," Physical Review Letters, vol. 64, no. 8, pp. 821-824, 1990.

[2] H. Serrano-Guerrero, C. Cruz-Hernandez, R. M. Lopez-Gutierrez, L. Cardoza-Avendano, and R. A. Chávez-Párez, "Chaotic synchronization in nearest-neighbor coupled Networks of 3D CNNs," Journal of Applied Research and Technology, vol. 11, no. 1, pp. 26-41, 2013.

[3] L. Jin, Y. Zhang, and L. Li, "One-to-many chaotic synchronization with application in wireless sensor network," IEEE Communications Letters, vol. 17, no. 9, pp. 1782-1785, 2013.

[4] V. Dronov, Application of Chaotic Synchronization and Controlling Chaos to Communications, Diss, 2005.

[5] S. Das, U. Halder, and D. Maity, "Chaotic dynamics in social foraging swarms-an analysis," IEEE Transactions on Systems, Man, and Cybernetics, Part B: Cybernetics, vol. 42, no. 4, pp. 1288-1293, 2012.

[6] Y. Yu and H.-X. Li, "Adaptive generalized function projective synchronization of uncertain chaotic systems," Nonlinear Analysis: Real World Applications, vol. 11, no. 4, pp. 2456-2464, 2010.

[7] G. M. Mahmoud, E. E. Mahmoud, and A. A. Arafa, "On projective synchronization of hyperchaotic complex nonlinear systems based on passive theory for secure communications," Physica Scripta, vol. 87, no. 5, Article ID 055002, 2013.

[8] J. Zhou, J.-a. Lu, and J. Lu, "Adaptive synchronization of an uncertain complex dynamical network," Institute of Electrical and Electronics Engineers Transactions on Automatic Control, vol. 51, no. 4, pp. 652-656, 2006.

[9] J. Sun, Q. Yin, and Y. Shen, "Compound synchronization for four chaotic systems of integer order and fractional order," $E P L$ (Europhysics Letters), vol. 106, no. 4, Article ID 40005, 2014.

[10] J. Sun, Y. Shen, X. Wang, and J. Chen, "Finite-time combinationcombination synchronization of four different chaotic systems with unknown parameters via sliding mode control," Nonlinear Dynamics, vol. 76, no. 1, pp. 383-397, 2014.

[11] C. Jiang and S. Liu, "Synchronization and antisynchronization of N-coupled complex permanent magnet synchronous motor systems with ring connection," Complexity, vol. 2017, Article ID 6743184, 15 pages, 2017.

[12] Q. Wei, X.-Y. Wang, and X.-P. Hu, "Hybrid function projective synchronization in complex dynamical networks," AIP Advances, vol. 4, no. 2, Article ID 027128, 2014.

[13] C. Li, Q. Chen, and T. Huang, "Coexistence of anti-phase and complete synchronization in coupled chen system via a single variable," Chaos, Solitons \& Fractals, vol. 38, no. 2, pp. 461-464, 2008.

[14] F. Nian and W. Liu, "Hybrid synchronization of heterogeneous chaotic systems on dynamic network," Chaos, Solitons \& Fractals, vol. 91, pp. 554-561, 2016.

[15] S. Wang, Y. Yu, and G. Wen, "Hybrid projective synchronization of time-delayed fractional order chaotic systems," Nonlinear Analysis: Hybrid Systems, vol. 11, pp. 129-138, 2014.

[16] C.-H. Chen, L.-J. Sheu, H.-K. Chen et al., "A new hyper-chaotic system and its synchronization," Nonlinear Analysis: Real World Applications, vol. 10, no. 4, pp. 2088-2096, 2009.

[17] M. Hu, Z. Xu, R. Zhang, and A. Hu, "Parameters identification and adaptive full state hybrid projective synchronization of chaotic (hyper-chaotic) systems," Physics Letters A, vol. 361, no. 3, pp. 231-237, 2007.
[18] X. Wu and H. Lu, "Hybrid synchronization of the general delayed and non-delayed complex dynamical networks via pinning control," Neurocomputing, vol. 89, pp. 168-177, 2012.

[19] Q. Song, J. Cao, and F. Liu, "Pinning-controlled synchronization of hybrid-coupled complex dynamical networks with mixed time-delays," International Journal of Robust and Nonlinear Control, vol. 22, no. 6, pp. 690-706, 2012.

[20] Y. Xu, W. Zhou, J. Fang, W. Sun, and L. Pan, "Adaptive synchronization of stochastic time-varying delay dynamical networks with complex-variable systems," Nonlinear Dynamics, vol. 81, no. 4, pp. 1717-1726, 2015.

[21] W. Yu, J. Cao, and J. Lü, "Global synchronization of linearly hybrid coupled networks with time-varying delay," SIAM Journal on Applied Dynamical Systems, vol. 7, no. 1, pp. 108-133, 2008.

[22] C. Jiang and S. Liu, "Generalized combination complex synchronization of new hyperchaotic complex Lü-like systems," Advances in Difference Equations, vol. 2015, no. 1, article no. 214, 2015.

[23] N. Cai, Y. Jing, and S. Zhang, "Generalized projective synchronization of different chaotic systems based on antisymmetric structure," Chaos, Solitons \& Fractals, vol. 42, no. 2, pp. 11901196, 2009.

[24] I. M. Kyprianidis and I. N. Stouboulos, "Chaotic synchronization of three coupled oscillators with ring connection," Chaos, Solitons \& Fractals, vol. 17, no. 2-3, pp. 327-336, 2003.

[25] Q. Song and J. Cao, "Synchronization and anti-synchronization for chaotic systems," Chaos, Solitons \& Fractals, vol. 33, no. 3, pp. 929-939, 2007.

[26] G. M. Mahmoud and E. E. Mahmoud, "Phase and antiphase synchronization of two identical hyperchaotic complex nonlinear systems," Nonlinear Dynamics, vol. 61, no. 1-2, pp. 141-152, 2010.

[27] S. B. Wang, X. Y. Wang, X. Y. Wang, and Y. F. Zhou, "Adaptive generalized combination complex synchronization of uncertain real and complex nonlinear systems," AIP Advances, vol. 6, no. 4, Article ID 045011, 2016.

[28] T. Dahms, J. Lehnert, and E. Schöll, "Cluster and group synchronization in delay-coupled networks," Physical Review E: Statistical, Nonlinear, and Soft Matter Physics, vol. 86, no. 1, part 2, Article ID 016202, 2012.

[29] M. Jiménez-Martín, J. Rodríguez-Laguna, O. D’Huys, J. de la Rubia, and E. Korutcheva, "Synchronization of fluctuating delay-coupled chaotic networks," Physical Review E: Statistical, Nonlinear, and Soft Matter Physics, vol. 95, no. 5, article 052210, 2017.

[30] Y. Sun, W. Li, and J. Ruan, “Generalized outer synchronization between complex dynamical networks with time delay and noise perturbation," Communications in Nonlinear Science and Numerical Simulation, vol. 18, no. 4, pp. 989-998, 2013.

[31] J. Lu, D. W. Ho, and J. Cao, "Synchronization in an array of nonlinearly coupled chaotic neural networks with delay coupling," International Journal of Bifurcation and Chaos, vol. 18, no. 10, pp. 3101-3111, 2008.

[32] X. Chen, J. Qiu, J. Cao, and H. He, "Hybrid synchronization behavior in an array of coupled chaotic systems with ring connection," Neurocomputing, vol. 173, pp. 1299-1309, 2016.

[33] V. Utkin and J. Shi, "Integral sliding mode in systems operating under uncertainty conditions," in Proceedings of the 35th IEEE Conference on Decision and Control, vol. 4, pp. 4591-4596, 1996. 


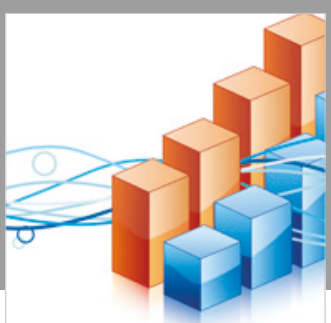

Advances in

Operations Research

\section{-n-m}
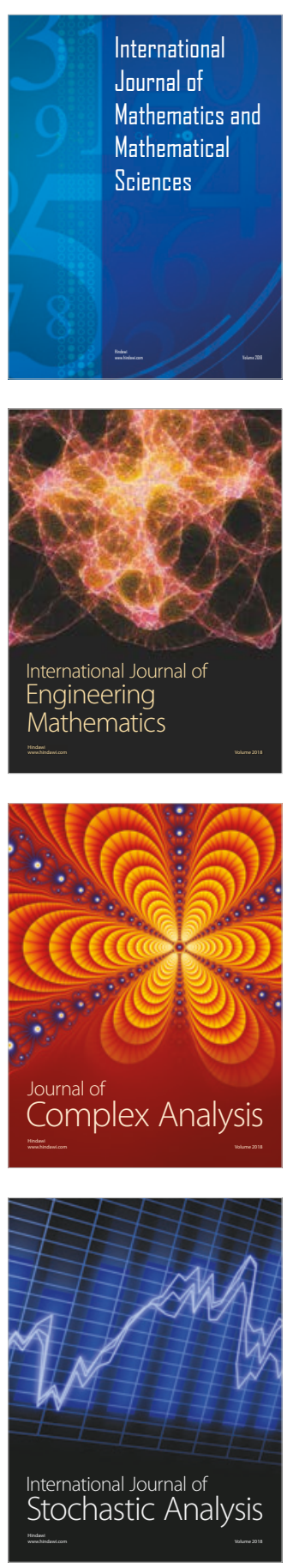
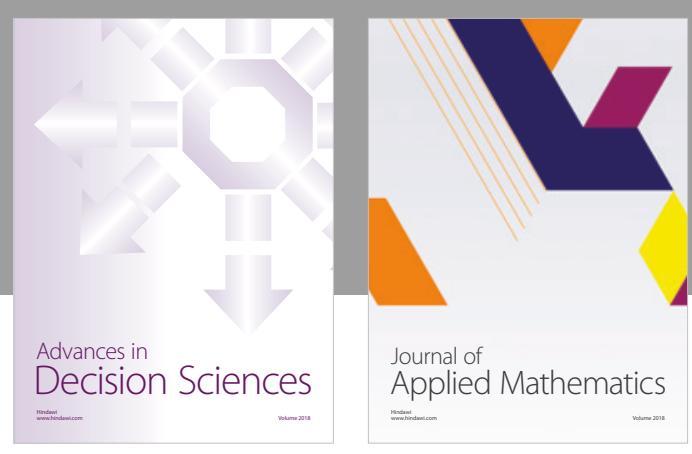

Journal of

Applied Mathematics
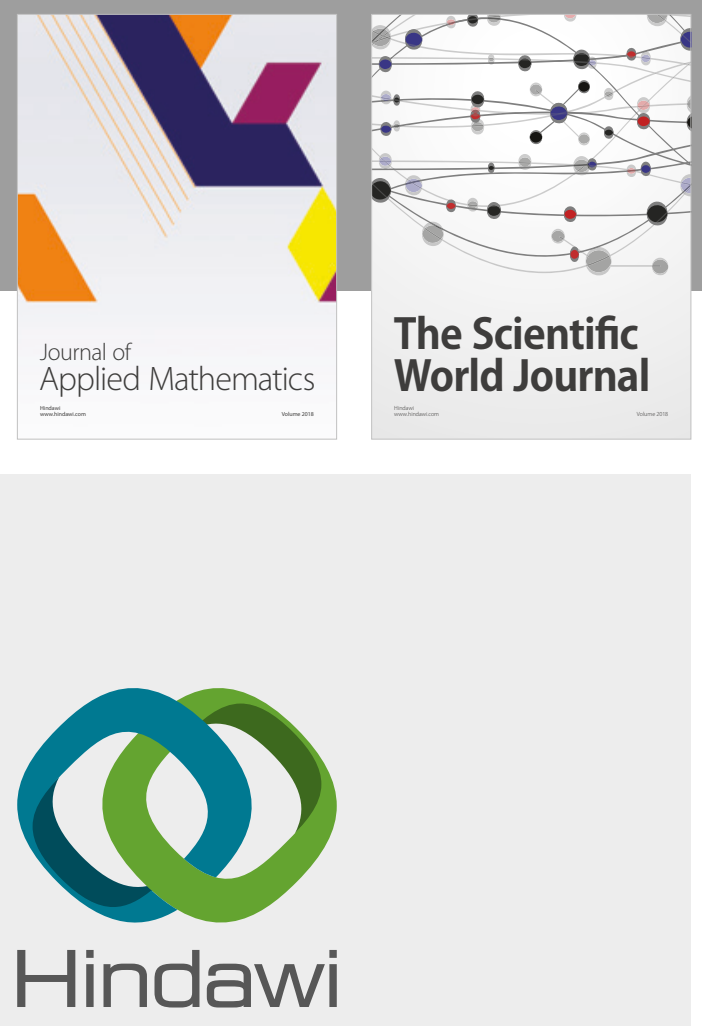

Submit your manuscripts at

www.hindawi.com

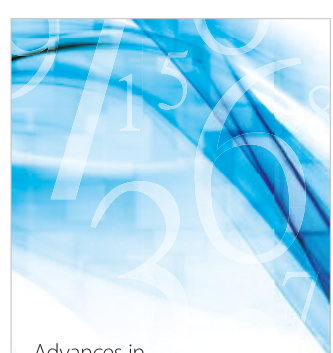

Advances in
Numerical Analysis
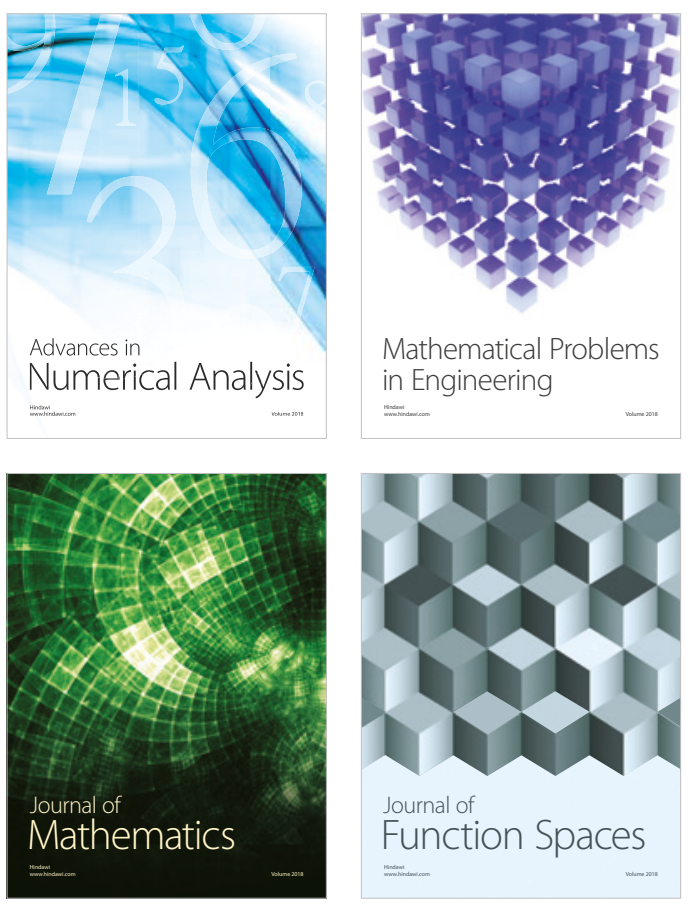

Mathematical Problems in Engineering

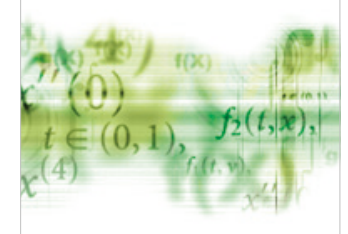

International Journal of

Differential Equations

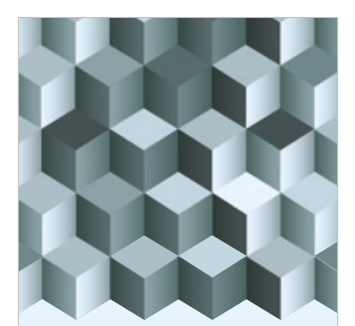

Journal of

Function Spaces

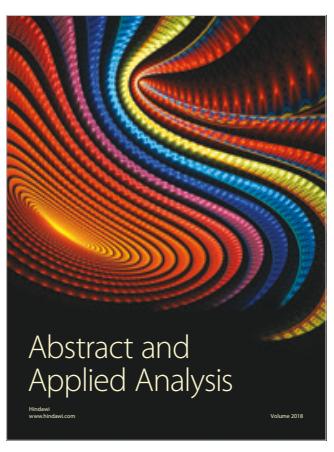

The Scientific

World Journal

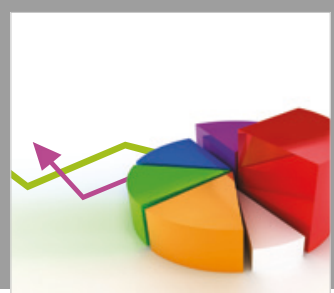

Journal of

Probability and Statistics
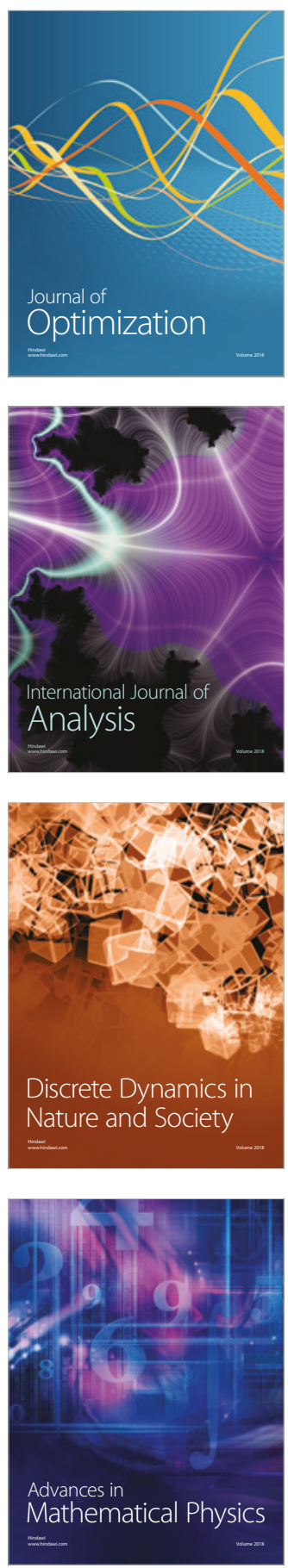